\begin{tabular}{|c|c|}
\hline Title & Confluent hy pergeometric systems associated with principal nilpotent $p$-tuples \\
\hline Author(s) & Saito, Mutsumi; Takeda, Hiroyasu \\
\hline Citation & $\begin{array}{l}\text { International journal of mathematics, 29(12), } 1850079 \\
\text { https://doi.org/10.1142/S0129167X 18500799 }\end{array}$ \\
\hline Issue Date & $2018-11$ \\
\hline Doc URL & http:/hdl.handle.net/2115//5980 \\
\hline Rights & $\begin{array}{l}\text { Electronic version of an article published as International Journal of Mathematics V ol. 29, No. 12, 2018, } 1850079 \\
\text { https://doi.org/10.1142/S0129167X 18500799 @ W orld Scientific Publishing Company } \\
\text { https:/Www.worldscientific.com/Worldscinet/jm }\end{array}$ \\
\hline Type & article (author version) \\
\hline File Information & SaitoT akeda23Revise.pdf \\
\hline
\end{tabular}

Instructions for use 


\title{
CONFLUENT HYPERGEOMETRIC SYSTEMS ASSOCIATED WITH PRINCIPAL NILPOTENT $p$-TUPLES
}

\author{
MUTSUMI SAITO AND HIROYASU TAKEDA
}

\begin{abstract}
Kimura and Takano showed that taking limits of regular elements of $\mathfrak{g l}(n)$ corresponds to the process of confluence of Aomoto-Gel'fand systems. We introduce a hypergeometric system associated with a principal nilpotent $p$-tuple, and, by using the principal nilpotent $p$-tuple, we directly deform a hypergeometric system of Gauss type into that of Airy type. Moreover we explicitly describe the deformation.
\end{abstract}

Mathematics Subject Classification (2010): 33C70 (primary), 16S32, 17B20 (secondary).

Keywords: Hypergeometric systems, confluence, principal nilpotent $p$-tuples.

\section{INTRODUCTION}

In this paper, we define and study a hypergeometric system associated with a principal nilpotent $p$-tuple. Its parameter space is the dual space of the centralizer of the principal nilpotent $p$-tuple.

Aomoto [1] and Gel'fand [3] independently defined a hypergeometric system of Gauss type, which is now called an Aomoto-Gel'fand system. The parameter space of an Aomoto-Gel'fand system is the dual space of a Cartan subalgebra of $\mathfrak{g l}(n, \mathbb{C})$. In [5], Gel'fand-Retahk-Serganova defined and studied a hypergeometric system of Airy type, which is a confluent version of an Aomoto-Gel'fand system. The parameter space of this system is the dual space of the Lie algebra of a Jordan Lie group (called a Jordan Lie subalgebra in [10]).

Inspired by the papers above, Kimura-Haraoka-Takano [7] introduced hypergeometric systems associated with regular elements of $\mathfrak{g l}(n, \mathbb{C})$. Their parameter spaces are the dual spaces of the centralizers $\mathfrak{z}_{\mathfrak{g} l(n, \mathbb{C})}(x)$ of the regular elements. (Recall that $x \in \mathfrak{g l}(n, \mathbb{C})$ is regular if $\operatorname{dim} \mathfrak{z}_{\mathfrak{g} \mathfrak{l}(n, \mathbb{C})}(x)=$ $n$.) These systems coincide with Aomoto-Gel'fand systems in the case where the regular elements are semisimple, and Gel'fand-Retahk-Serganova's systems in the case where the regular elements are nilpotent. For the

Date: April 28, 2018. 
other regular elements, we obtain systems in-between. For example, we obtain Gauss, Kummer, Bessel, Hermite-Weber, and Airy for $n=4$. Furthermore, in [9], Kimura-Takano explained the process of confluence between their hypergeometric systems by taking limits of regular elements.

There exist many Lie subalgebras which are limits of Cartan subalgebras but not the centralizers of regular elements (cf. [10]). It is natural to ask if the systems of differential equations associated with these Lie subalgebras are obtained as the limits of systems of Gauss type.

In this paper, we introduce principal nilpotent $p$-tuples. Principal nilpotent 1-tuples are regular nilpotent elements, and principal nilpotent 2-tuples (pairs) were introduced by Ginzburg [6]. He also classified principal nilpotent pairs of type $A_{n}$ by using Young diagrams. Principal nilpotent pairs of the other classical types were classified by ElashviliPanyushev [2] and R. Yu [11]. The centralizer of a principal nilpotent $p$-tuple is a limit of Cartan subalgebras as follows:

Lemma 1.1 (Lemma 5.1). Let $\left(e_{1}, \ldots, e_{p}\right)$ be a principal nilpotent $p$ tuple, and let $\mathfrak{h}$ be the Cartan subalgebra corresponding to $\left(e_{1}, \ldots, e_{p}\right)$. Set $\boldsymbol{e}:=e_{1}+\cdots+e_{p}$ and $\boldsymbol{e}(\tau):=\exp \left(\frac{\tau-1}{\tau} \boldsymbol{e}\right) \in G L(n)$. Then we have

$$
\lim _{\tau \rightarrow 0} \operatorname{Ad}(\boldsymbol{e}(\tau)) \mathfrak{h}=\mathfrak{z}_{\mathfrak{g l} l}(n, \mathbb{C})\left(e_{1}, \ldots, e_{p}\right):=\cap_{i=1}^{p} \mathfrak{z}_{\mathfrak{g l}}\left(e_{i}\right) .
$$

In this paper, we take the limit of the action of $\boldsymbol{e}(\tau)$ to deform hypergeometric systems of Gauss type into the system associated with $\left(e_{1}, \ldots, e_{p}\right)$, along with the corresponding integrands of integral representations of solutions.

This paper is organized as follows. In Section 2 we construct hypergeometric systems whose parameter spaces are $n$-dimension abelian Lie subalgebra of $\mathfrak{g l}(n)$ inspired by the definition of hypergeometric systems defined by Gel'fand et al. In Section 3 we introduce principal nilpotent $p$-tuples. In Section 4 we exhibit an integral representation of a solution to a hypergeometric system associated with a principal nilpotent $p$-tuple. In Section 5 we consider the deformation of integrands of integral representations of solutions. In Section 6 we study the action of the normalizer of the abelian subalgebra generated by a principal nilpotent $p$-tuple, to specialize the parameters of the corresponding hypergeometric systems, as in [8]. In Section 7, as one example of the systems, we consider the hypergeometric system associated with a principal nilpotent pair corresponding to the Young diagram $(n-1,1)$. We show that this system is generically holonomic, and the solutions coincide with generalized Airy functions. 


\section{Hypergeometric Systems À LA Gel'FAnd}

Throughout this paper, set $G=G L(n, \mathbb{C})($ or $G L(V))$ and $\mathfrak{g}=$ $\mathfrak{g l}(n, \mathbb{C})$ (or $\mathfrak{g l}(V))$, where $V$ is complex $n$-dimensional vector space.

In this section, we recall the definitions of hypergeometric systems of Gauss type [3] and of Airy type [5] defined by Gel'fand et al.

Let $G=G L(n, \mathbb{C})$, and $N=\left\{\left[\begin{array}{cc}I_{m} & B \\ O & D\end{array}\right] \in G \mid D \in G L(n-m)\right\}$.

Then we have the morphism

$$
G \ni g \mapsto g\left[\begin{array}{c}
I_{m} \\
O
\end{array}\right] \in G / N=\left\{z \in M_{n, m}(\mathbb{C}) \mid \operatorname{rank}(z)=m\right\}=: Z .
$$

Let $\Theta_{Z}$ denote the sheaf of vector fields on $Z$. Naturally we have a Lie algebra homomorphism $\partial: \mathfrak{g}=\mathfrak{g l}(n, \mathbb{C}) \ni a \mapsto \partial_{a} \in \Theta_{Z}(Z)$,

$$
\left(\partial_{a}(f)\right)(z)=\frac{d}{d t}_{\mid t=0} f(\exp (-t a) z) \quad\left(a \in \mathfrak{g}, f \in \mathcal{O}_{Z}, z \in Z\right) .
$$

Let $\mathfrak{a}$ be an $n$-dimensional abelian subalgebra of $\mathfrak{g}$. For a given $\alpha \in \mathfrak{a}^{*}$, we consider a hypergeometric system $M_{\mathfrak{a}, \alpha}$ defined on $Z$ :

$$
\begin{gathered}
\mathcal{D}_{Z} /\left(\sum_{p, q=1}^{n} \sum_{j_{1}, j_{2}=1}^{m} \mathcal{D}_{Z}\left(\partial_{p, j_{1}} \partial_{q, j_{2}}-\partial_{p, j_{2}} \partial_{q, j_{1}}\right)\right. \\
\quad+\sum_{i, j=1}^{m} \mathcal{D}_{Z}\left(\sum_{k=1}^{n} z_{k, i} \partial_{k, j}+\delta_{i, j}\right) \\
\quad+\sum_{a \in \mathfrak{a}} \mathcal{D}_{Z}\left(\partial_{a}-\alpha(a)\right) .
\end{gathered}
$$

When we take the Cartan subalgebra consisting of diagonal matrices as $\mathfrak{a}$, the system $M_{\mathfrak{a}, \alpha}$ coincides with the Gel'fand's hypergeometric system [3]. When we take the Jordan Lie subalgebra as a:

$$
\mathfrak{a}=\left\langle e^{i} \mid i=0,1, \ldots, n-1\right\rangle,
$$

where $e=\sum_{i=1}^{n-1} E_{i+1, i}$, and $E_{i, j}$ is the matrix with $(i, j)$-entry 1 and with 0 for the other entries, the system $M_{\mathfrak{a}, \alpha}$ is the generalized Airy system [5].

Lemma 2.1. For $a=\left[a_{i j}\right] \in \mathfrak{g}=\mathfrak{g l}(n, \mathbb{C})$,

$$
\partial_{a}=-\sum_{k, l, s} a_{k, s} z_{s, l} \partial_{k, l}
$$

In particular, for $i, j \leq n$,

$$
\partial_{E_{i, j}}=-\sum_{l=1}^{m} z_{j, l} \partial_{i, l}
$$


Proof.

$$
\begin{aligned}
\left(\partial_{a} f\right)(Z) & =\frac{d}{d t} \mid t=0 \\
& =\sum_{k, l}\left(\frac{d}{d t}{ }_{\mid t=0}[\exp (-t a) Z)\right. \\
& \left.=\sum_{k, l}\left(\left.\frac{d}{d t}\right|_{t=0}[Z-t a) Z\right]_{k, l}\right) \partial_{k, l} f(Z) \\
& =-\sum_{k, l}[a Z]_{k, l} \partial_{k, l} f(Z) \\
& \left.\left.=-\sum_{k, l, s} a_{k, s} z_{s, l}\right]_{k, l}\right) \partial_{k, l} f(Z)
\end{aligned}
$$

\section{PRINCIPAL NilPotent $p$-TUPLES}

In this section, we introduce principal nilpotent $p$-tuples, which were defined by Ginzburg [6] for $p=2$.

Let $\mathfrak{h}$ be a Cartan subalgebra of $\mathfrak{g}$. From now on, we suppose that there exist $h_{1}, \ldots, h_{p} \in \mathfrak{h}$ and $e_{1}, \ldots, e_{p} \in \mathfrak{g}$ satisfying

(1) $\left[h_{i}, e_{j}\right]=\delta_{i j} e_{j} \quad(1 \leq i, j \leq p)$,

(2) $\left[e_{i}, e_{j}\right]=0 \quad(1 \leq i, j \leq p)$,

(3) Let $\mathfrak{a}:=\left\langle e^{\boldsymbol{l}} \mid \boldsymbol{l} \in \mathbb{N}^{p}\right\rangle$, where $e^{\boldsymbol{l}}=\prod_{i=1}^{p} e_{i}^{l_{i}}$ for $\boldsymbol{l}=\left(l_{1}, \ldots, l_{p}\right) \in$ $\mathbb{N}^{p}$. Then the centralizer $\mathfrak{z}_{\mathfrak{g}}(\mathfrak{a})$ equals $\mathfrak{a}$, and $\operatorname{dim} \mathfrak{a}=n$.

We say such $p$-tuple $\left(e_{1}, \ldots, e_{p}\right)$ to be principal (cf. [6] $(p=2$ case)).

Clearly each $e_{i}$ is nilpotent, and

$$
\left[h_{i}, e^{\boldsymbol{l}}\right]=l_{i} e^{\boldsymbol{l}}
$$

Put

$$
L:=\left\{\boldsymbol{l} \in \mathbb{N}^{p} \mid e^{\boldsymbol{l}} \neq 0\right\} .
$$

Then $\left\{e^{\boldsymbol{l}} \mid \boldsymbol{l} \in L\right\}$ forms a basis of $\mathfrak{a}$.

The following lemma can be proved in the same way as in the proof of $[6$, Theorem 1.2 (ii)].

Lemma 3.1. $\mathfrak{z}_{\mathfrak{g}}\left(h_{1}, \ldots, h_{p}\right):=\cap_{i=1}^{p} \mathfrak{z}_{\mathfrak{g}}\left(h_{i}\right)=\mathfrak{h}$.

Example 3.2. Let $p=1$. Let $e$ be a regular nilpotent element. Since there exists a semisimple element $H$ with $[H, e]=2 e$, putting $h=\frac{1}{2} H$, we see that $h$ and $e$ satisfy the above conditions. For example, we can take $h=\sum_{i=1}^{n} i E_{i, i}, e=\sum_{i=1}^{n-1} E_{i+1, i}$. Then $\mathfrak{a}$ is the Jordan Lie subalgebra (2). 
Since $h_{1}, \ldots, h_{p}$ are commuting semisimple elements, they are simultaneously diagonalizable. Let $A=\left\{\boldsymbol{a}=\left(a_{1}, \ldots, a_{p}\right)\right\}$ be the set of joint eigenvalues, and let $\left\{v_{\boldsymbol{a}} \mid \boldsymbol{a} \in A\right\}$ be a basis of $V$ consisting of joint eigenvectors:

$$
h_{i} v_{\boldsymbol{a}}=a_{i} v_{\boldsymbol{a}} \quad(i=1, \ldots, p ; \boldsymbol{a} \in A) .
$$

Multiplying $v_{\boldsymbol{a}}$ by some constants if necessary, we may suppose that

$$
e^{\boldsymbol{l}} v_{\boldsymbol{a}}=v_{\boldsymbol{a}+\boldsymbol{l}} \quad(\boldsymbol{a}, \boldsymbol{a}+\boldsymbol{l} \in A, \boldsymbol{l} \in L)
$$

by (3). Throughout this paper, we suppose that there exists $\boldsymbol{a}(0) \in A$ such that

$$
A=\boldsymbol{a}(0)+L .
$$

With respect to the basis $\left\{v_{\boldsymbol{a}}\right\}, h_{i}$ and $e_{i}$ are represented by

$$
\sum_{\boldsymbol{a} \in A} a_{i} E_{\boldsymbol{a}, \boldsymbol{a}}, \quad \sum_{\boldsymbol{a}+1_{i}, \boldsymbol{a} \in A} E_{\boldsymbol{a}+1_{i}, \boldsymbol{a}},
$$

respectively. More generally $h^{l}$ and $e^{l}$ are respectively represented by

$$
\sum_{\boldsymbol{a} \in A} \boldsymbol{a}^{\boldsymbol{l}} E_{\boldsymbol{a}, \boldsymbol{a}}, \quad \sum_{\boldsymbol{a}+\boldsymbol{l}, \boldsymbol{a} \in A} E_{\boldsymbol{a}+\boldsymbol{l}, \boldsymbol{a}},
$$

where $h^{\boldsymbol{l}}=h_{1}^{l_{1}} \cdots h_{p}^{l_{p}}$ and $\boldsymbol{a}^{\boldsymbol{l}}=a_{1}^{l_{1}} \cdots a_{p}^{l_{p}}$ for $\boldsymbol{l}=\left(l_{1}, \ldots, l_{p}\right)$.

Example 3.3. Let $n=4, p=2$, and let

$$
\begin{array}{ll}
e_{1}=\left[\begin{array}{llll}
0 & 0 & 0 & 0 \\
1 & 0 & 0 & 0 \\
0 & 1 & 0 & 0 \\
0 & 0 & 0 & 0
\end{array}\right], \quad e_{2}=\left[\begin{array}{llll}
0 & 0 & 0 & 0 \\
0 & 0 & 0 & 0 \\
0 & 0 & 0 & 0 \\
1 & 0 & 0 & 0
\end{array}\right], \\
h_{1}=\left[\begin{array}{llll}
0 & 0 & 0 & 0 \\
0 & 1 & 0 & 0 \\
0 & 0 & 2 & 0 \\
0 & 0 & 0 & 0
\end{array}\right], \quad h_{2}=\left[\begin{array}{llll}
0 & 0 & 0 & 0 \\
0 & 0 & 0 & 0 \\
0 & 0 & 0 & 0 \\
0 & 0 & 0 & 1
\end{array}\right] .
\end{array}
$$

Then $\left(e_{1}, e_{2}\right)$ is a principal nilpotent pair,

$$
A=L=\{(0,0),(1,0),(2,0),(0,1)\}=\begin{array}{|l|l|}
\hline(0,0) & (1,0)(2,0) \\
\hline(0,1) &
\end{array}
$$

and

$$
\mathfrak{a}=\left\langle I_{4}, e_{1}, e_{1}^{2}, e_{2}\right\rangle
$$

Remark 3.4. As in Example 3.3, we can associate $L$ with a $p$-dimensional Young diagram of size $n$. For example, $L$ in Example 3.2 is associated with $\square\left[\begin{array}{l|l|}\hline & \\ \hline\end{array}\right.$.

In [7], for a Young diagram $\lambda$, Kimura, Haraoka and Takano considered a confluent hypergeometric system of type $\lambda$. Note that the 
subalgebra associated with Young diagram $\lambda$ in this paper is different from the one in [7]. For example, with Young diagram $\square \square$, as in Example 3.3 we associate the subalgebra

$$
\left\{\left[\begin{array}{cccc}
c_{0} & 0 & 0 & 0 \\
c_{1} & c_{0} & 0 & 0 \\
c_{2} & c_{1} & c_{0} & 0 \\
c_{3} & 0 & 0 & c_{0}
\end{array}\right] \mid c_{0}, \ldots, c_{3} \in \mathbb{C}\right\},
$$

but the subalgebra

$$
\left\{\left[\begin{array}{cccc}
c_{0} & 0 & 0 & 0 \\
c_{1} & c_{0} & 0 & 0 \\
c_{2} & c_{1} & c_{0} & 0 \\
0 & 0 & 0 & c_{3}
\end{array}\right] \mid c_{0}, \ldots, c_{3} \in \mathbb{C}\right\}
$$

is associated in [7].

Lemma 3.5. The matrix $\left[\boldsymbol{a}^{\boldsymbol{l}}\right]_{\boldsymbol{l} \in L, \boldsymbol{a} \in A}$ is non-singular.

Proof. The set of rows is $L$, and the set of columns is $A=\boldsymbol{a}(0)+L$. Put

$$
D(\boldsymbol{a}(0)+L):=\operatorname{det}\left(\left[\boldsymbol{a}^{\boldsymbol{l}}\right]_{\boldsymbol{l} \in L ; \boldsymbol{a} \in \boldsymbol{a}(0)+L}\right) .
$$

When $p=1$,

$$
D(\boldsymbol{a}(0)+L)=\prod_{k<l}((\boldsymbol{a}(0)+l)-(\boldsymbol{a}(0)+k))=\prod_{k<l} \pm(l-k) \neq 0
$$

by Vandermonde.

Now we consider the general case. In the matrix $\left[\boldsymbol{a}^{\boldsymbol{l}}\right]$, we add $-\boldsymbol{a}(0)_{p^{-}}$ times of the $\boldsymbol{l}$-th row to the $\boldsymbol{l}+1_{p}$-th row. Then the $(\boldsymbol{l}, \boldsymbol{a})$-component becomes $\boldsymbol{a}^{\boldsymbol{l}}-\boldsymbol{a}(0)_{p} \boldsymbol{a}^{\boldsymbol{l}-1_{p}}=\boldsymbol{a}^{\boldsymbol{l}-1_{p}}\left(a_{p}-a(0)_{p}\right)$ for $l_{p}>0$; remains $\boldsymbol{a}^{\boldsymbol{l}}$ for $l_{p}=0$. In particular, the columns $\boldsymbol{a}$ with $a_{p}=a(0)_{p}$ have components 0 in the rows $\boldsymbol{l}$ with $l_{p}>0$. Hence

$$
D(\boldsymbol{a}(0)+L)=D\left(\boldsymbol{a}(0)+L^{\prime}\right) D\left(\boldsymbol{a}(0)+1_{p}+L^{\prime \prime}\right) \prod_{\boldsymbol{a}=\boldsymbol{a}(0)+\boldsymbol{l} ; l_{p}>0}\left(a_{p}-a(0)_{p}\right),
$$

where

$$
\begin{aligned}
L^{\prime} & =\left\{\boldsymbol{l} \in L \mid l_{p}=0\right\}, \\
L^{\prime \prime} & =\left\{\boldsymbol{l}-1_{p} \mid \boldsymbol{l} \in L, l_{p}>0\right\} .
\end{aligned}
$$

Then by repeating the similar discussion for $D\left(\boldsymbol{a}(0)+1_{p}+L^{\prime \prime}\right)$, we have

$$
D(\boldsymbol{a}(0)+L)=\prod_{i} D\left(\boldsymbol{a}(0)+i 1_{p}+L_{i}\right) \prod_{\boldsymbol{a}=\boldsymbol{a}(0)+\boldsymbol{l} ; l_{p}>0}\left(a_{p}-a(0)_{p}\right)^{l_{p}}
$$

where $L_{i}=\left\{l-i 1_{p} \mid l \in L, l_{p}=i\right\}$. Hence by the induction we have proved $D(\boldsymbol{a}(0)+L) \neq 0$. 
Corollary 3.6. $\left\langle h^{l} \mid \boldsymbol{l} \in L\right\rangle=\mathfrak{h}$.

Proof. Since $h^{l}$ acts as $\operatorname{diag}\left(\boldsymbol{a}^{\boldsymbol{l}}: \boldsymbol{a} \in A\right)$, the $n$ elements $h^{\boldsymbol{l}}(\boldsymbol{l} \in L)$ are linearly independent by Lemma 3.5. Hence the assertion holds.

Let $\left(e_{1}, \ldots, e_{p}\right)$ be a principal nilpotent $p$-tuple. Take $B \subseteq A$ with $|B|=m$, and let $V_{B}=\oplus_{\boldsymbol{a} \in B} \mathbb{C} v_{\boldsymbol{a}}$. Set

$$
\begin{aligned}
G & =G L(V), \quad \mathfrak{g}=\mathfrak{g l}(V)=\operatorname{End}(V), \\
N & =N_{B}=\left\{g \in G \mid g_{\mid V_{B}}=i d_{V_{B}}\right\}, \quad Z=G / N_{B} .
\end{aligned}
$$

Let $B=\left\{\boldsymbol{b}_{1}, \ldots, \boldsymbol{b}_{m}\right\}$, and we write $j$ for $\boldsymbol{b}_{j}$. Then, by Lemma 2.1, for $\mathfrak{a}=\left\langle e^{\boldsymbol{l}} \mid \boldsymbol{l} \in L\right\rangle$ and $\alpha \in \mathfrak{a}^{*}$ the system $M_{\mathfrak{a}, \alpha}$ (1) is rewritten as follows:

$$
\begin{aligned}
& \left(\frac{\partial}{\partial z_{\boldsymbol{a}, j_{1}}} \frac{\partial}{\partial z_{\boldsymbol{b}, j_{2}}}-\frac{\partial}{\partial z_{\boldsymbol{a}, j_{2}}} \frac{\partial}{\partial z_{\boldsymbol{b}, j_{1}}}\right) \Phi=0 \quad\left(\boldsymbol{a}, \boldsymbol{b} \in A ; 1 \leq j_{1}, j_{2} \leq m\right), \\
& \left(\sum_{\boldsymbol{a} \in A} z_{\boldsymbol{a}, j_{1}} \frac{\partial}{\partial z_{\boldsymbol{a}, j_{2}}}+\delta_{j_{1} j_{2}}\right) \Phi=0 \quad\left(1 \leq j_{1}, j_{2} \leq m\right), \\
& \left(\sum_{\boldsymbol{a}, \boldsymbol{a}+\boldsymbol{l} \in A} \sum_{j=1}^{m} z_{\boldsymbol{a}, j} \frac{\partial}{\partial z_{\boldsymbol{a}+\boldsymbol{l}, j}}+\alpha\left(e^{\boldsymbol{l}}\right)\right) \Phi=0 \quad(\boldsymbol{l} \in L) .
\end{aligned}
$$

Remark 3.7. A different $L$ defines a different subalgebra $\mathfrak{a}$, hence a different system. A system in [5] corresponds to the Jordan Lie subalgebra (2) (cf. Example 3.2). Systems $M_{\mathfrak{a}, \alpha}$ which are also systems in [7] are only the systems in [5], since the semisimple part of our subalgebra $\mathfrak{a}=\left\langle e^{\boldsymbol{l}} \mid \boldsymbol{l} \in L\right\rangle$ is 1-dimensional as in Remark 3.4.

\section{Integral REPRESENTATIONS}

In this section, after recalling an integral representation of a solution to the system for $\mathfrak{h}$, we exhibit an integral reprensentation of a solution to the system associated with a principal nilpotent $p$-tuple.

Set

$$
d t:=\sum_{i=1}^{m}(-1)^{i-1} t_{i} d t_{1} \wedge \cdots \wedge{\widehat{d t_{i}}} \wedge \cdots \wedge d t_{m} .
$$

\section{Proposition 4.1 ([3]).}

$$
\psi(\alpha, z):=\int \prod_{\boldsymbol{a} \in A}\left(\sum_{j=1}^{m} z_{\boldsymbol{a}, j} t_{j}\right)^{\alpha_{\boldsymbol{a}}} d t
$$

is a solution to the system $M_{\mathfrak{h},-\alpha}$, where $\alpha \in \mathfrak{h}^{*}$ is defined by $\alpha\left(E_{\boldsymbol{a}, \boldsymbol{a}}\right)=$ $\alpha_{\boldsymbol{a}}(\boldsymbol{a} \in A)$. 
Proof. Let $g \in G L(m)$. Then

$$
\begin{aligned}
\psi(\alpha, z g) & =\int \prod_{\boldsymbol{a} \in A}\left(\sum_{j=1}^{m} \sum_{k=1}^{m} z_{\boldsymbol{a}, k} g_{k, j} t_{j}\right)^{\alpha_{\boldsymbol{a}}} d t \\
& =\int \prod_{\boldsymbol{a} \in A}\left(\sum_{k=1}^{m} z_{\boldsymbol{a}, k}\left(\sum_{j=1}^{m} g_{k, j} t_{j}\right)\right)^{\alpha_{\boldsymbol{a}}} d t .
\end{aligned}
$$

Put

$$
T_{k}:=\sum_{j=1}^{m} g_{k, j} t_{j}
$$

Then $t_{j}=\sum_{k=1}^{m}\left(g^{-1}\right)_{j, k} T_{k}$ and $d t=\operatorname{det}(g)^{-1} d T$. Hence

$$
\psi(\alpha, z g)=\operatorname{det}(g)^{-1} \int \prod_{\boldsymbol{a} \in A}\left(\sum_{k=1}^{m} z_{\boldsymbol{a}, k} T_{k}\right)^{\alpha \boldsymbol{a}} d T=\operatorname{det}(g)^{-1} \psi(\alpha, z),
$$

and $\psi$ satisfies $(7)$.

We have

$$
\begin{aligned}
\frac{\partial}{\partial z_{\boldsymbol{b}, k}}\left(\prod_{\boldsymbol{a} \in A}\left(\sum_{j=1}^{m} z_{\boldsymbol{a}, j} t_{j}\right)^{\alpha_{\boldsymbol{a}}}\right) & \left.\left.=\prod_{\boldsymbol{a} \neq \boldsymbol{b}}\left(\sum_{j=1}^{m} z_{\boldsymbol{a}, j} t_{j}\right)^{\alpha_{\boldsymbol{a}}}\right) \frac{\partial}{\partial z_{\boldsymbol{b}, k}}\left(\sum_{j=1}^{m} z_{\boldsymbol{b}, j} t_{j}\right)^{\alpha_{\boldsymbol{b}}}\right) \\
& \left.=\alpha_{\boldsymbol{b}} t_{k} \prod_{\boldsymbol{a} \neq \boldsymbol{b}}\left(\sum_{j=1}^{m} z_{\boldsymbol{a}, j} t_{j}\right)^{\alpha_{\boldsymbol{a}}}\right)\left(\sum_{j=1}^{m} z_{\boldsymbol{b}, j} t_{j}\right)^{\alpha_{\boldsymbol{b}}-1} .
\end{aligned}
$$

Hence

$$
\left.\left(\frac{\partial}{\partial z_{\boldsymbol{b}, k}} \frac{\partial}{\partial z_{\boldsymbol{c}, l}}-\frac{\partial}{\partial z_{\boldsymbol{b}, l}} \frac{\partial}{\partial z_{\boldsymbol{c}, k}}\right)\left(\prod_{\boldsymbol{a} \in A}\left(\sum_{j=1}^{m} z_{\boldsymbol{a}, j} t_{j}\right)^{\alpha_{\boldsymbol{a}}}\right)\right)=0
$$

and

$$
\sum_{k=1}^{m} z_{\boldsymbol{b}, k} \frac{\partial}{\partial z_{\boldsymbol{b}, k}}\left(\prod_{\boldsymbol{a} \in A}\left(\sum_{j=1}^{m} z_{\boldsymbol{a}, j} t_{j}\right)^{\alpha_{\boldsymbol{a}}}\right)=\alpha_{\boldsymbol{b}} \prod_{\boldsymbol{a} \in A}\left(\sum_{j=1}^{m} z_{\boldsymbol{a}, j} t_{j}\right)^{\alpha_{\boldsymbol{a}}} .
$$

Hence $\psi$ is a solution to the system $M_{\mathfrak{h},-\alpha}$.

As in [5], we introduce the polynomials $\theta_{\boldsymbol{k}}$; let

$$
\sum_{\boldsymbol{l} \in \mathbb{N}^{p}} b_{\boldsymbol{l}} T^{\boldsymbol{l}}=\exp \left(\sum_{\boldsymbol{k} \in \mathbb{N}^{p} \backslash\{\mathbf{0}\}} \theta_{\boldsymbol{k}}(b) T^{\boldsymbol{k}}\right)
$$

where $b_{\mathbf{0}}=1$.

\section{Lemma 4.2.}

$$
\sum_{\boldsymbol{j} \geq \boldsymbol{l}} b_{\boldsymbol{j}-\boldsymbol{l}} \frac{\partial \theta_{\boldsymbol{k}}}{\partial b_{\boldsymbol{j}}}= \begin{cases}1 & (\boldsymbol{k}=\boldsymbol{l}) \\ 0 & (\boldsymbol{k} \neq \boldsymbol{l})\end{cases}
$$

Proof. By definition, in $\mathbb{C}\left[b_{\boldsymbol{l}}\left(\boldsymbol{l} \in \mathbb{N}^{p} \backslash\{\mathbf{0}\}\right)\right]\left[\left[T_{1}, \ldots T_{p}\right]\right]$,

$$
\log \left(\sum_{\boldsymbol{i} \in \mathbb{N}^{p}} b_{\boldsymbol{i}} T^{\boldsymbol{i}}\right)=\sum_{\boldsymbol{k} \in \mathbb{N}^{p} \backslash\{\mathbf{0}\}} \theta_{\boldsymbol{k}} T^{\boldsymbol{k}} .
$$


By executing $\frac{\partial}{\partial b_{j}}$, we have

$$
\frac{T^{j}}{\left(\sum_{\boldsymbol{i} \geq \mathbf{0}} b_{\boldsymbol{i}} T^{\boldsymbol{i}}\right)}=\sum_{\boldsymbol{k} \geq \mathbf{0}} \frac{\partial \theta_{\boldsymbol{k}}}{\partial b_{\boldsymbol{j}}} T^{\boldsymbol{k}}
$$

Hence

$$
\frac{T^{j-l}}{\left(\sum_{\boldsymbol{i} \geq \mathbf{0}} b_{\boldsymbol{i}} T^{\boldsymbol{i}}\right)}=\sum_{\boldsymbol{k} \ngtr \mathbf{0}} \frac{\partial \theta_{\boldsymbol{k}}}{\partial b_{j}} T^{\boldsymbol{k}-\boldsymbol{l}}
$$

and

$$
1=\frac{\sum_{\boldsymbol{j} \geq \boldsymbol{l}} b_{\boldsymbol{j}-\boldsymbol{l}} T^{\boldsymbol{j}-\boldsymbol{l}}}{\left(\sum_{\boldsymbol{i} \geq \mathbf{0}} b_{\boldsymbol{i}} T^{\boldsymbol{i}}\right)}=\sum_{\boldsymbol{k} \ngtr \mathbf{0}} \sum_{\boldsymbol{j} \geq \boldsymbol{l}} b_{\boldsymbol{j}-\boldsymbol{l}} \frac{\partial \theta_{\boldsymbol{k}}}{\partial b_{\boldsymbol{j}}} T^{\boldsymbol{k}-\boldsymbol{l}},
$$

Therefore we have the assertion.

Proposition 4.3. For $\boldsymbol{l} \in L$, let $b_{\boldsymbol{l}}=\sum_{j=1}^{m} z_{\boldsymbol{a}(0)+\boldsymbol{l}, j} t_{j}, b_{\boldsymbol{l}}^{\prime}:=b_{\boldsymbol{l}} / b_{\mathbf{0}}$, and

$$
\phi(\alpha, z, t):=b_{\mathbf{0}}^{\alpha_{0}} \exp \left(\sum_{\boldsymbol{k} \in L_{+}} \alpha_{\boldsymbol{k}} \theta_{\boldsymbol{k}}\left(b^{\prime}\right)\right),
$$

where $L_{+}=L \backslash\{\mathbf{0}\}$. Then

$$
\sum_{\boldsymbol{i} \geq \boldsymbol{l}} \sum_{j=1}^{m} z_{\boldsymbol{a}(0)+\boldsymbol{i}-\boldsymbol{l}, j} \frac{\partial}{\partial z_{\boldsymbol{a}(0)+\boldsymbol{i}, j}} \phi=\alpha_{\boldsymbol{l}} \phi .
$$

Proof. Let $\boldsymbol{l} \neq \mathbf{0}$. Then

$$
\begin{aligned}
\sum_{\boldsymbol{i} \ngtr \boldsymbol{l}} \sum_{j=1}^{m} z_{\boldsymbol{a}(0)+\boldsymbol{i}-\boldsymbol{l}, j} \frac{\partial}{\partial z_{\boldsymbol{a}(0)+\boldsymbol{i}, j}} \phi & =\sum_{\boldsymbol{i} \ngtr \boldsymbol{l}} \sum_{j=1}^{m} z_{\boldsymbol{a}(0)+\boldsymbol{i}-\boldsymbol{l}, j} \phi \sum_{\boldsymbol{k} \in L_{+}} \alpha_{\boldsymbol{k}} \frac{\partial}{\partial z_{\boldsymbol{a}(0)+\boldsymbol{i}, j}} \theta_{\boldsymbol{k}}\left(b^{\prime}\right) \\
& =\sum_{\boldsymbol{i} \ngtr \boldsymbol{l}} \sum_{j=1}^{m} z_{\boldsymbol{a}(0)+\boldsymbol{i}-\boldsymbol{l}, j} \phi \sum_{\boldsymbol{k} \in L_{+}} \alpha_{\boldsymbol{k}} \frac{\partial \theta_{\boldsymbol{k}}}{\partial b_{\boldsymbol{i}}} t_{j} \\
& =\phi \sum_{\boldsymbol{k} \in L_{+}} \alpha_{\boldsymbol{k}} \sum_{\boldsymbol{i} \ngtr \boldsymbol{l}} b_{\boldsymbol{i}-\boldsymbol{l}} \frac{\partial \theta_{\boldsymbol{k}}}{\partial b_{\boldsymbol{i}}}=\alpha_{\boldsymbol{l}} \phi
\end{aligned}
$$

by Lemma 4.2. Clearly $\phi\left(\alpha, e^{\tau} z, t\right)=e^{\alpha_{0} \tau} \phi(\alpha, z, t)$. Hence we have the equation for $\boldsymbol{l}=\mathbf{0}$.

Proposition 4.4 ([5] for $p=1)$.

$$
\psi(\alpha, z):=\int \phi(\alpha, z, t) d t
$$

is a solution to the system $M_{\mathfrak{a},-\alpha}$, where $\mathfrak{a}=\left\langle e^{\boldsymbol{l}} \mid \boldsymbol{l} \in L\right\rangle$, and $\alpha \in \mathfrak{a}^{*}$ is defined by $\alpha\left(e^{\boldsymbol{l}}\right)=\alpha_{\boldsymbol{l}}(\boldsymbol{l} \in L)$.

Proof. By Proposition 4.3, $\psi$ satisfies (8). As in the proof of Proposition 4.1 , it is easy to see

$$
\psi(\alpha, z g)=\operatorname{det}(g)^{-1} \psi(\alpha, z),
$$


and $\psi$ satisfies (7).

We have, for $\boldsymbol{i} \neq \mathbf{0}$,

$$
\begin{aligned}
\frac{\partial}{\partial z_{\boldsymbol{a}(0)+\boldsymbol{i}, j}} \phi & =\phi \cdot \frac{\partial}{\partial z_{\boldsymbol{a}(0)+\boldsymbol{i}, j}}\left(\sum_{\boldsymbol{k}} \alpha_{\boldsymbol{k}} \theta_{\boldsymbol{k}}\right) \\
& =\phi \cdot \sum_{\boldsymbol{k}} \alpha_{\boldsymbol{k}} \frac{\partial \theta_{\boldsymbol{k}}}{\partial z_{\boldsymbol{a}(0)+\boldsymbol{i}, j}} \\
& =\phi \cdot \sum_{\boldsymbol{k}} \alpha_{\boldsymbol{k}} \frac{\partial \theta_{\boldsymbol{k}}}{\partial b_{\boldsymbol{i}}} t_{j} .
\end{aligned}
$$

We also have

$$
\begin{aligned}
\frac{\partial}{\partial z_{\boldsymbol{a}(0), j}} \phi & =\alpha_{\mathbf{0}} b_{\mathbf{0}}^{-1} t_{j} \phi+\phi \cdot \sum_{\boldsymbol{k}} \alpha_{\boldsymbol{k}} \frac{\partial \theta_{\boldsymbol{k}}}{\partial z_{\boldsymbol{a}(0), j}} \\
& =\alpha_{\mathbf{0}} b_{\mathbf{0}}^{-1} t_{j} \phi-\phi \cdot \sum_{\boldsymbol{k}, \boldsymbol{i}} \alpha_{\boldsymbol{k}} \frac{\partial \theta_{\boldsymbol{k}}}{\partial b_{\boldsymbol{i}}^{\prime}} \frac{b_{\boldsymbol{i}}}{b_{\mathbf{0}}^{2}} t_{j} \\
& =b_{\mathbf{0}}^{-1} t_{j} \phi\left(\alpha_{\mathbf{0}}-\sum_{\boldsymbol{k}, \boldsymbol{i}} \alpha_{\boldsymbol{k}} b_{\boldsymbol{i}} \frac{\partial \theta_{\boldsymbol{k}}}{\partial b_{\boldsymbol{i}}}\right) .
\end{aligned}
$$

From the above equations,

$$
\begin{aligned}
& \frac{\partial^{2}}{\partial z_{\boldsymbol{a}(0)+\boldsymbol{i}_{1}, j_{1}} \partial z_{\boldsymbol{a}(0)+\boldsymbol{i}_{2}, j_{2}}} \phi \\
= & \frac{\partial}{\partial z_{\boldsymbol{a}(0)+\boldsymbol{i}_{1}, j_{1}}}\left(\phi \cdot \sum_{\boldsymbol{k}} \alpha_{\boldsymbol{k}} \frac{\partial \theta_{\boldsymbol{k}}}{\partial b_{\boldsymbol{i}_{2}}} t_{j_{2}}\right) \\
= & \phi\left(\left(\sum_{\boldsymbol{k}^{\prime}} \alpha_{\boldsymbol{k}^{\prime}} \frac{\partial \theta_{\boldsymbol{k}^{\prime}}}{\partial b_{\boldsymbol{i}_{1}}}\right)\left(\sum_{\boldsymbol{k}} \alpha_{\boldsymbol{k}} \frac{\partial \theta_{\boldsymbol{k}}}{\partial b_{\boldsymbol{i}_{2}}}\right)+\sum_{\boldsymbol{k}} \alpha_{\boldsymbol{k}} \frac{\partial^{2} \theta_{\boldsymbol{k}}}{\partial b_{\boldsymbol{i}_{1}} \partial b_{\boldsymbol{i}_{2}}}\right) t_{j_{1}} t_{j_{2}}
\end{aligned}
$$

for $\boldsymbol{i}_{1}, \boldsymbol{i}_{2} \neq \mathbf{0}, \boldsymbol{i}_{1} \neq \boldsymbol{i}_{2}, j_{1} \neq j_{2}$, and

$$
\begin{aligned}
& \frac{\partial^{2}}{\partial z_{\boldsymbol{a}(0), j_{1}} \partial z_{\boldsymbol{a}(0)+\boldsymbol{i}_{2}, j_{2}}} \phi \\
= & \frac{\partial}{\partial z_{\boldsymbol{a}(0), j_{1}}}\left(\phi \cdot \sum_{\boldsymbol{k}} \alpha_{\boldsymbol{k}} \frac{\partial \theta_{\boldsymbol{k}}}{\partial b_{\boldsymbol{i}_{2}}} t_{j_{2}}\right) \\
= & b_{\mathbf{0}}^{-1} \phi\left(\left(\alpha_{\mathbf{0}}-\sum_{\boldsymbol{k}^{\prime}, \boldsymbol{i}} \alpha_{\boldsymbol{k}^{\prime}} b_{\boldsymbol{i}} \frac{\partial \theta_{\boldsymbol{k}^{\prime}}}{\partial b_{\boldsymbol{i}}}\right)\left(\sum_{\boldsymbol{k}} \alpha_{\boldsymbol{k}} \frac{\partial \theta_{\boldsymbol{k}}}{\partial b_{\boldsymbol{i}_{2}}}\right)-\sum_{\boldsymbol{k}, \boldsymbol{i}} \alpha_{\boldsymbol{k}} b_{\boldsymbol{i}} \frac{\partial^{2} \theta_{\boldsymbol{k}}}{\partial b_{\boldsymbol{i}} \partial b_{\boldsymbol{i}_{2}}}\right) t_{j_{1}} t_{j_{2}}
\end{aligned}
$$

for $\boldsymbol{i}_{2} \neq 0, j_{1} \neq j_{2}$. Then we see that

$$
\frac{\partial^{2}}{\partial z_{\boldsymbol{a}(0)+\boldsymbol{i}_{1}, j_{1}} \partial z_{\boldsymbol{a}(0)+\boldsymbol{i}_{2}, j_{2}}} \phi
$$


is symmetric in $j_{1}$ and $j_{2}$. Hence

$$
\left(\frac{\partial^{2}}{\partial z_{\boldsymbol{a}(0)+\boldsymbol{i}_{1}, j_{1}} \partial z_{\boldsymbol{a}(0)+\boldsymbol{i}_{2}, j_{2}}}-\frac{\partial^{2}}{\partial z_{\boldsymbol{a}(0)+\boldsymbol{i}_{1}, j_{2}} \partial z_{\boldsymbol{a}(0)+\boldsymbol{i}_{2}, j_{1}}}\right) \phi=0 .
$$

\section{Deformation}

In the previous section, we saw an integral representation of a solution to $M_{\mathfrak{h},-\alpha}$ (Proposition 4.1) and that to $M_{\mathfrak{a},-\alpha}$ (Proposition 4.4). In this section, we naturally deform the integrand of the former to that of the latter (Theorem 5.6), which is the main theorem of this paper.

Recall that $e_{i}$ is represented by

$$
\sum_{\boldsymbol{a}+1_{i}, \boldsymbol{a} \in A} E_{\boldsymbol{a}+1_{i}, \boldsymbol{a}}
$$

and $e^{l}$ is represented by

$$
\sum_{a+l, a \in A} E_{a+l, a}
$$

Recall that for $\mathfrak{h}=\left\langle h^{l}\right\rangle$ the system $M_{\mathfrak{h},-\alpha}$, where $\alpha \in \mathfrak{h}^{*}$ is defined by $\alpha\left(E_{\boldsymbol{a}, \boldsymbol{a}}\right)=\alpha_{\boldsymbol{a}}(\boldsymbol{a} \in A)$, is the following:

$$
\begin{aligned}
& \left(\frac{\partial}{\partial z_{\boldsymbol{a}, i}} \frac{\partial}{\partial z_{\boldsymbol{b}, j}}-\frac{\partial}{\partial z_{\boldsymbol{a}, j}} \frac{\partial}{\partial z_{\boldsymbol{b}, i}}\right) \Phi=0 \quad(\boldsymbol{a}, \boldsymbol{b} \in A ; 1 \leq i, j \leq m) \\
& \left(\sum_{\boldsymbol{a} \in A} z_{\boldsymbol{a}, i} \frac{\partial}{\partial z_{\boldsymbol{a}, j}}+\delta_{i j}\right) \Phi=0 \quad(1 \leq i, j \leq m) \\
& \left(\sum_{k=1}^{m} z_{\boldsymbol{a}, k} \frac{\partial}{\partial z_{\boldsymbol{a}, k}}-\alpha_{\boldsymbol{a}}\right) \Phi=0 \quad(\boldsymbol{a} \in A) .
\end{aligned}
$$

Let

$$
\alpha_{\boldsymbol{l}}=\sum_{\boldsymbol{a} \in A} \boldsymbol{a}^{\boldsymbol{l}} \alpha_{\boldsymbol{a}}
$$

The equations (13) are equivalent to

$$
\left(\sum_{\boldsymbol{a} \in A} \boldsymbol{a}^{\boldsymbol{l}} \sum_{k=1}^{m} z_{\boldsymbol{a}, k} \frac{\partial}{\partial z_{\boldsymbol{a}, k}}-\alpha_{\boldsymbol{l}}\right) \Phi=0 \quad(\boldsymbol{l} \in L),
$$

since $h^{l}$ corresponds to

$$
\sum_{a \in A} a^{l} E_{a, a}
$$

The equation (14) can be rewritten as

$$
\left(\partial_{h^{l}}+\alpha_{\boldsymbol{l}}\right) \Phi=0 \quad(\boldsymbol{l} \in L)
$$


Set $\boldsymbol{e}:=e_{1}+\cdots+e_{p}$, and consider $\boldsymbol{e}(\tau):=\exp \left(\frac{\tau-1}{\tau} \boldsymbol{e}\right) \in G=G L(n)$. Then $\boldsymbol{e}(1)=1$.

Lemma 5.1. (1) $\lim _{\tau \rightarrow 0} \operatorname{Ad}(\boldsymbol{e}(\tau))(\tau h)^{M}=e^{M}$ for all $M \in \mathbb{N}^{p}$.

(2) $\lim _{\tau \rightarrow 0} \operatorname{Ad}(\boldsymbol{e}(\tau)) \mathfrak{h}=\mathfrak{a}$.

Proof. Since $\left[\boldsymbol{e}, h_{i}\right]=-e_{i}$, we have

$$
\operatorname{Ad}(\boldsymbol{e}(\tau))\left(h_{i}\right)=\exp \operatorname{ad}\left(\frac{\tau-1}{\tau} \boldsymbol{e}\right)\left(h_{i}\right)=h_{i}+\frac{1-\tau}{\tau} e_{i} .
$$

Since $\operatorname{Ad}(\boldsymbol{e}(\tau))$ is an automorphism of an associative algebra,

$$
\operatorname{Ad}(\boldsymbol{e}(\tau))\left((\tau h)^{\boldsymbol{l}}\right)=\prod_{i=1}^{p}\left(\tau h_{i}+(1-\tau) e_{i}\right)^{l_{i}} .
$$

Hence we see the assertion (1), and hence (2).

We have the left multiplication map $L_{\boldsymbol{e}(\tau)}$ on $Z=G / N$. In general,

$$
L_{g} \circ \partial_{a} \circ L_{g}^{-1}=\partial_{\operatorname{Ad}(g) a} \quad(g \in G, a \in \mathfrak{g}) .
$$

Lemma 5.2.

$$
L_{\boldsymbol{e}(\tau)} \cdot z_{\boldsymbol{a}, j}=\sum_{\boldsymbol{k} \geq 0} \frac{1}{\boldsymbol{k} !}\left(\frac{1-\tau}{\tau}\right)^{|\boldsymbol{k}|} z_{\boldsymbol{a}-\boldsymbol{k}, j} .
$$

Proof. By an abuse of notation, we denote a general matrix in $Z$ by $Z$ again. By definition,

$$
\left(L_{\boldsymbol{e}(\tau)} \cdot z_{\boldsymbol{a}, j}\right)(Z)=z_{\boldsymbol{a}, j}\left(\boldsymbol{e}(\tau)^{-1} Z\right) .
$$

We have

$$
\boldsymbol{e}(\tau)^{-1} Z=\sum_{\boldsymbol{k} \geq 0} \frac{1}{\boldsymbol{k} !}\left(\frac{1-\tau}{\tau}\right)^{|\boldsymbol{k}|} \boldsymbol{e}^{\boldsymbol{k}} Z
$$

Hence

$$
\left(\boldsymbol{e}(\tau)^{-1} Z\right)_{\boldsymbol{a}, j}=\sum_{\boldsymbol{k} \geq 0} \frac{1}{\boldsymbol{k} !}\left(\frac{1-\tau}{\tau}\right)^{|\boldsymbol{k}|} Z_{\boldsymbol{a}-\boldsymbol{k}, j}
$$

\section{Corollary 5.3.}

$$
L_{\boldsymbol{e}(\tau)} \cdot \partial_{\boldsymbol{a}, j}=\sum_{\boldsymbol{b} \geq \boldsymbol{a}} \frac{1}{(\boldsymbol{b}-\boldsymbol{a}) !}\left(\frac{\tau-1}{\tau}\right)^{|\boldsymbol{b}-\boldsymbol{a}|} \partial_{\boldsymbol{b}, j} .
$$

Proof. We have

$$
\begin{aligned}
\left(\left(L_{\boldsymbol{e}(\tau)} \cdot \partial_{\boldsymbol{a}, j}\right) f\right)(Z) & =\left(\left(L_{\boldsymbol{e}(\tau)} \circ \partial_{\boldsymbol{a}, j} \circ L_{\boldsymbol{e}(\tau)}^{-1}\right) f\right)(Z) \\
& =L_{\boldsymbol{e}(\tau)} \cdot\left(\partial_{\boldsymbol{a}, j}(f(\boldsymbol{e}(\tau) Z))\right) .
\end{aligned}
$$


By Lemma 5.2,

$$
\frac{\partial(\boldsymbol{e}(\tau) Z)_{\boldsymbol{b}, i}}{\partial z_{\boldsymbol{a}, j}}= \begin{cases}\delta_{i, j} \frac{1}{(\boldsymbol{b}-\boldsymbol{a}) !}\left(\frac{\tau-1}{\tau}\right)^{|\boldsymbol{b}-\boldsymbol{a}|} & (\boldsymbol{b}-\boldsymbol{a} \geq 0) \\ 0 & \text { (otherwise) }\end{cases}
$$

Hence

$$
\begin{aligned}
\partial_{\boldsymbol{a}, j}(f(\boldsymbol{e}(\tau) Z)) & =\sum_{(\boldsymbol{b}, i)}\left(\partial_{\boldsymbol{b}, i} f\right)(\boldsymbol{e}(\tau) Z) \frac{\partial(\boldsymbol{e}(\tau) Z)_{\boldsymbol{b}, i}}{\partial z_{\boldsymbol{a}, j}} \\
& =\sum_{\boldsymbol{b} \geq \boldsymbol{a}}\left(\partial_{\boldsymbol{b}, j} f\right)(\boldsymbol{e}(\tau) Z) \frac{1}{(\boldsymbol{b}-\boldsymbol{a}) !}\left(\frac{\tau-1}{\tau}\right)^{|\boldsymbol{b}-\boldsymbol{a}|}
\end{aligned}
$$

and the assertion holds.

\section{Corollary 5.4.}

$$
L_{\boldsymbol{e}(\tau)} \cdot\left(\sum_{\boldsymbol{k} \geq \mathbf{0}} \frac{1}{\boldsymbol{k} !}\left(\frac{1-\tau}{\tau}\right)^{|\boldsymbol{k}|} \partial_{\boldsymbol{a}+\boldsymbol{k}, j}\right)=\partial_{\boldsymbol{a}, j} .
$$

Proof. Clear from Corollary 5.3.

We consider the system $M_{\operatorname{Ad}(\boldsymbol{e}(\tau)) \mathfrak{h},-\alpha(\tau)}$, where $\alpha(\tau) \in(\operatorname{Ad}(\boldsymbol{e}(\tau)) \mathfrak{h})^{*}$ is defined by $(\alpha(\tau))\left(\operatorname{Ad}(\boldsymbol{e}(\tau)) h^{l}\right)=\frac{\alpha_{l}}{\tau^{|l|}}$. Hence the equations (15) become

$$
\left(\partial_{\operatorname{Ad}(e(\tau)) h^{l}}+\frac{\alpha_{\boldsymbol{l}}}{\tau^{|\boldsymbol{l}|}}\right) \Phi=0 \quad(\boldsymbol{l} \in L)
$$

Put

$$
\phi_{\tau}(\alpha, z)=\prod_{\boldsymbol{a} \in A}\left(L_{\boldsymbol{e}(\tau)} \cdot b_{\boldsymbol{a}-\boldsymbol{a}(0)}\right)^{\alpha_{\boldsymbol{a}}} .
$$

Then $\phi_{1}(\alpha, z)$ coincides with the integrand in Proposition 4.1. We have

$$
\begin{aligned}
\partial_{\operatorname{Ad}(e(\tau)) h^{l}} \phi_{\tau}(\alpha, z) & =L_{\boldsymbol{e}(\tau)} \circ \partial_{h^{l}} \circ L_{\boldsymbol{e}(\tau)}^{-1} \phi_{\tau}(\alpha, z) \\
& =L_{\boldsymbol{e}(\tau)} \circ \partial_{h^{l}} \phi(\alpha, z) \\
& =-L_{\boldsymbol{e}(\tau)} \sum_{\boldsymbol{a} \in A} \boldsymbol{a}^{\boldsymbol{l}} \alpha_{\boldsymbol{a}} \phi(\alpha, z) \\
& =-\sum_{\boldsymbol{a} \in A} \boldsymbol{a}^{\boldsymbol{l}} \alpha_{\boldsymbol{a}} \phi_{\tau}(\alpha, z) .
\end{aligned}
$$

Let $\left[\beta_{\boldsymbol{a}}^{\boldsymbol{l}}\right]$ be the inverse of $\left[\boldsymbol{a}^{\boldsymbol{l}}\right]$. Then for $\alpha_{\boldsymbol{a}}=\sum_{\boldsymbol{l}} \beta_{\boldsymbol{a}}^{\boldsymbol{l}} \alpha_{\boldsymbol{l}} / \tau^{|\boldsymbol{l}|}$

$$
\partial_{\operatorname{Ad}(e(\tau)) h^{l}} \phi_{\tau}(\alpha, z)=-\frac{\alpha_{\boldsymbol{l}}}{\tau^{|l|}} \phi_{\tau}(\alpha, z) .
$$

Hence we have the following proposition: 
Proposition 5.5. Let $\left[\beta_{\boldsymbol{a}}^{\boldsymbol{l}}\right]$ be the inverse of $\left[\boldsymbol{a}^{\boldsymbol{l}}\right]$, and let

$$
\phi_{\tau}(\alpha, z)=\prod_{\boldsymbol{l} \in L}\left(\prod_{\boldsymbol{a} \in A}\left(L_{\boldsymbol{e}(\tau)} \cdot b_{\boldsymbol{a}-\boldsymbol{a}(0)}\right)^{\beta_{\boldsymbol{a}}^{\boldsymbol{a}}}\right)^{\alpha_{\boldsymbol{l}} / \tau^{|\boldsymbol{l}|}} .
$$

Then

$$
\int \phi_{\tau}(\alpha, z) d t
$$

satisfies the system $M_{\operatorname{Ad}(\boldsymbol{e}(\tau)) \mathfrak{h},-\alpha(\tau)}$ :

$$
\begin{aligned}
& \left(\frac{\partial}{\partial z_{\boldsymbol{a}, i}} \frac{\partial}{\partial z_{\boldsymbol{b}, j}}-\frac{\partial}{\partial z_{\boldsymbol{a}, j}} \frac{\partial}{\partial z_{\boldsymbol{b}, i}}\right) \Phi=0 \quad(\boldsymbol{a}, \boldsymbol{b} \in A ; i, j \in B) \\
& \left(\sum_{\boldsymbol{a} \in A} z_{\boldsymbol{a}, i} \frac{\partial}{\partial z_{\boldsymbol{a}, j}}+\delta_{i j}\right) \Phi=0 \quad(i, j \in B) \\
& \left(\partial_{\operatorname{Ad}(\boldsymbol{e}(\tau)) h^{\boldsymbol{l}}}+\frac{\alpha_{\boldsymbol{l}}}{\tau^{|\boldsymbol{l}|}}\right) \Phi=0 \quad(\boldsymbol{l} \in L),
\end{aligned}
$$

where the compatibility condition is $\alpha_{\mathbf{0}}=-m$.

Proof. The compatibility condition is

$$
\sum_{\alpha \in A} \alpha_{\boldsymbol{a}}=-m
$$

We have

$$
\begin{aligned}
\sum_{\boldsymbol{a} \in A} \alpha_{\boldsymbol{a}} & =\sum_{\boldsymbol{a} \in A} \sum_{\boldsymbol{l} \in L} \beta_{\boldsymbol{a}}^{\boldsymbol{l}} \alpha_{\boldsymbol{l}} / \tau^{|\boldsymbol{l}|} \\
& =\sum_{\boldsymbol{l} \in L} \sum_{\boldsymbol{a} \in A} \boldsymbol{a}^{\mathbf{0}} \beta_{\boldsymbol{a}}^{\boldsymbol{l}} \alpha_{\boldsymbol{l}} / \tau^{|\boldsymbol{l}|} \\
& =\alpha_{\mathbf{0}} / \tau^{\mathbf{0}}=\alpha_{\mathbf{0}} .
\end{aligned}
$$

Since $\sum_{\boldsymbol{a} \in A} \boldsymbol{a}^{\mathbf{0}} \beta_{\boldsymbol{a}}^{\boldsymbol{l}} \alpha_{\boldsymbol{l}} / \tau^{\boldsymbol{l}}=\delta_{\boldsymbol{l}, \mathbf{0}} \alpha_{\mathbf{0}}$, we have

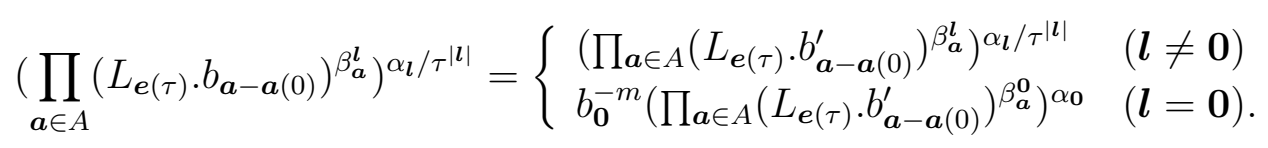

Hence

$$
\phi_{\tau}(\alpha, z)=b_{\mathbf{0}}^{-m} \prod_{\boldsymbol{l} \in L}\left(\prod_{\boldsymbol{a} \in A}\left(L_{\boldsymbol{e}(\tau)} \cdot b_{\boldsymbol{a}-\boldsymbol{a}(0)}^{\prime}\right)^{\beta_{\boldsymbol{a}}^{\boldsymbol{l}}}\right)^{\alpha_{\boldsymbol{l}} / \tau^{|l|}} .
$$

Note that $\phi_{\tau}=L_{\boldsymbol{e}(\tau)} \cdot \phi_{1}$. Put

$$
T_{A}:=\left\langle\frac{\partial}{\partial z_{\boldsymbol{a}, i}} \frac{\partial}{\partial z_{\boldsymbol{b}, j}}-\frac{\partial}{\partial z_{\boldsymbol{a}, j}} \frac{\partial}{\partial z_{\boldsymbol{b}, i}} \mid \boldsymbol{a}, \boldsymbol{b} \in A, 1 \leq i<j \leq m\right\rangle .
$$

Since $T_{A} \phi_{1}=0$, we have

$$
\begin{aligned}
\left(L_{\boldsymbol{e}(\tau)} \cdot T_{A}\right) \phi_{\tau} & =\left(L_{\boldsymbol{e}(\tau)} \circ T_{A} \circ L_{\boldsymbol{e}(\tau)}^{-1}\right)\left(L_{\boldsymbol{e}(\tau)} \phi_{1}\right) \\
& =\left(L_{\boldsymbol{e}(\tau)} \circ T_{A}\right)\left(\phi_{1}\right)=0 .
\end{aligned}
$$


By Corollary 5.4, we have $L_{\boldsymbol{e}(\tau)} \cdot T_{A} \supset T_{A}$, and hence $\phi_{\tau}$ satisfies (17).

Put

$$
\varphi_{\tau}(\alpha, z):=\left(\frac{\tau}{1-\tau}\right)^{\sum_{\boldsymbol{a} \in A} \alpha_{\boldsymbol{a}}|\boldsymbol{a}-\boldsymbol{a}(0)|} \prod_{\boldsymbol{a} \in A}((\boldsymbol{a}-\boldsymbol{a}(0)) !)^{\alpha_{\boldsymbol{a}}} \phi_{\tau}(\alpha, z) .
$$

Then $\int \varphi_{\tau}(\alpha, z)$ is also a solution of the system $M_{\operatorname{Ad}(\boldsymbol{e}(\tau)) \mathfrak{h},-\alpha(\tau)}$. The following theorem corresponds to [9, Theorem 6.3].

\section{Theorem 5.6.}

$$
\varphi_{\tau}(\alpha, z) \rightarrow b_{\mathbf{0}}^{-m} \exp \left(\sum_{\boldsymbol{l} \in L} \alpha_{\boldsymbol{l}} \theta_{\boldsymbol{l}}\left(b^{\prime}\right)\right)=\phi(\alpha, z, t) \quad(\tau \rightarrow 0) .
$$

Proof. By definition,

$$
\begin{aligned}
\varphi_{\tau}(\alpha, z) & =b_{\mathbf{0}}^{-m} \prod_{\boldsymbol{l} \in L}\left(\prod_{\boldsymbol{a} \in A}\left(\sum_{\boldsymbol{k} \geq 0} \frac{(\boldsymbol{a}-\boldsymbol{a}(0)) !}{\boldsymbol{k} !}\left(\frac{\tau}{1-\tau}\right)^{|\boldsymbol{a}-\boldsymbol{a}(0)-\boldsymbol{k}|} b_{\boldsymbol{a}-\boldsymbol{a}(0)-\boldsymbol{k}}^{\prime}\right)^{\beta_{\boldsymbol{a}}^{\boldsymbol{l}}}\right)^{\alpha_{\boldsymbol{l}} / \tau^{|\boldsymbol{l}|}} \\
& =b_{\mathbf{0}}^{-m} \prod_{\boldsymbol{l} \in L}\left(\prod_{\boldsymbol{a} \in A}\left(\sum_{\boldsymbol{a}(0) \leq \boldsymbol{d} \leq \boldsymbol{a}} \frac{(\boldsymbol{a}-\boldsymbol{a}(0)) !}{(\boldsymbol{a}-\boldsymbol{d}) !}\left(\frac{\tau}{1-\tau}\right)^{|\boldsymbol{d}-\boldsymbol{a}(0)|} b_{\boldsymbol{d}-\boldsymbol{a}(0)}^{\prime}\right)^{\beta_{\boldsymbol{a}}^{\boldsymbol{l}}}\right)^{\alpha_{\boldsymbol{l}} / \tau^{|\boldsymbol{l}|}} .
\end{aligned}
$$

For $\boldsymbol{k}^{\prime} \leq \boldsymbol{k}$, define $c_{\boldsymbol{k}^{\prime}, \boldsymbol{k}} \in \mathbb{C}$ by

$$
\prod_{i=1}^{p}\left(X_{i}-\boldsymbol{a}(0)_{i}\right)\left(X_{i}-\boldsymbol{a}(0)_{i}-1\right) \cdots\left(X_{i}-\boldsymbol{a}(0)_{i}-k_{i}+1\right)=\sum_{\mathbf{0} \leq \boldsymbol{k}^{\prime} \leq \boldsymbol{k}} c_{\boldsymbol{k}^{\prime}, \boldsymbol{k}} X^{\boldsymbol{k}^{\prime}} .
$$

Note that $c_{\boldsymbol{k}, \boldsymbol{k}}=1$. Then for $\boldsymbol{a}(0) \leq \boldsymbol{d} \leq \boldsymbol{a}$

$$
\frac{(\boldsymbol{a}-\boldsymbol{a}(0)) !}{(\boldsymbol{a}-\boldsymbol{d}) !}=\sum_{\mathbf{0} \leq \boldsymbol{k}^{\prime} \leq \boldsymbol{d}-\boldsymbol{a}(0)} c_{\boldsymbol{k}^{\prime}, \boldsymbol{d}-\boldsymbol{a}(0)} \boldsymbol{a}^{\boldsymbol{k}^{\prime}}
$$

Hence

$$
\begin{aligned}
& \prod_{\boldsymbol{a} \in A}\left(\sum_{\boldsymbol{a}(0) \leq \boldsymbol{d} \leq \boldsymbol{a}} \frac{(\boldsymbol{a}-\boldsymbol{a}(0)) !}{(\boldsymbol{a}-\boldsymbol{d}) !}\left(\frac{\tau}{1-\tau}\right)^{|\boldsymbol{d}-\boldsymbol{a}(0)|} b_{\boldsymbol{d}-\boldsymbol{a}(0)}^{\prime}\right)^{\beta_{\boldsymbol{a}}^{\boldsymbol{l}}} \\
= & \prod_{\boldsymbol{a} \in A}\left(\sum_{\boldsymbol{a}(0) \leq \boldsymbol{d} \leq \boldsymbol{a}} \sum_{\mathbf{0} \leq \boldsymbol{k}^{\prime} \leq \boldsymbol{d}-\boldsymbol{a}(0)} c_{\boldsymbol{k}^{\prime}, \boldsymbol{d}-\boldsymbol{a}(0)} \boldsymbol{a}^{\boldsymbol{k}^{\prime}}\left(\frac{\tau}{1-\tau}\right)^{|\boldsymbol{d}-\boldsymbol{a}(0)|} b_{\boldsymbol{d}-\boldsymbol{a}(0)}^{\prime}\right)^{\beta_{\boldsymbol{a}}^{\boldsymbol{l}}} .
\end{aligned}
$$

We have by Lemma 5.7 below

$$
\begin{gathered}
\sum_{\mathbf{0} \leq \boldsymbol{k}^{\prime} \leq \boldsymbol{k} \leq \boldsymbol{a}-\boldsymbol{a}(0)} c_{\boldsymbol{k}^{\prime}, \boldsymbol{k}} \boldsymbol{a}^{\boldsymbol{k}^{\prime}}\left(\frac{\tau}{1-\tau}\right)^{|\boldsymbol{k}|} b_{\boldsymbol{k}}^{\prime} \\
=\exp \left(\sum_{\mathbf{0} \leq \boldsymbol{k}^{\prime} \leq \boldsymbol{k}} \theta_{\boldsymbol{k}^{\prime}, \boldsymbol{k}}\left(\left\{c_{\boldsymbol{s}^{\prime}, \boldsymbol{s}} b_{\boldsymbol{s}}^{\prime}\right\}\right) \boldsymbol{a}^{\boldsymbol{k}^{\prime}}\left(\frac{\tau}{1-\tau}\right)^{|\boldsymbol{k}|}\right),
\end{gathered}
$$


where in the statement of Lemma 5.7 we put $S_{i}=\frac{\tau}{1-\tau}$ for $i=1,2, \ldots, p$. Hence

$$
\begin{aligned}
& \prod_{\boldsymbol{a} \in A}\left(\sum_{\boldsymbol{a}(0) \leq \boldsymbol{d} \leq \boldsymbol{a}} \frac{(\boldsymbol{a}-\boldsymbol{a}(0)) !}{(\boldsymbol{a}-\boldsymbol{d}) !}\left(\frac{\tau}{1-\tau}\right)^{|\boldsymbol{d}-\boldsymbol{a}(0)|} b_{\boldsymbol{d}-\boldsymbol{a}(0)}^{\prime}\right)^{\beta_{\boldsymbol{a}}^{\boldsymbol{l}}} \\
& =\prod_{\boldsymbol{a} \in A} \exp \left(\sum_{\mathbf{0} \leq \boldsymbol{k}^{\prime} \leq \boldsymbol{k}} \theta_{\boldsymbol{k}^{\prime}, \boldsymbol{k}}\left(\left\{c_{\boldsymbol{s}^{\prime}, \boldsymbol{s}} b_{\boldsymbol{s}}^{\prime}\right\}\right) \boldsymbol{a}^{\boldsymbol{k}^{\prime}}\left(\frac{\tau}{1-\tau}\right)^{|\boldsymbol{k}|}\right)^{\beta_{\boldsymbol{a}}^{\boldsymbol{l}}} \\
& =\exp \left(\sum_{\boldsymbol{a} \in A} \sum_{\mathbf{0} \leq \boldsymbol{k}^{\prime} \leq \boldsymbol{k}} \beta_{\boldsymbol{a}}^{\boldsymbol{l}} \theta_{\boldsymbol{k}^{\prime}, \boldsymbol{k}}\left(\left\{c_{\boldsymbol{s}^{\prime}, \boldsymbol{s}} b_{\boldsymbol{s}}^{\prime}\right\}\right) \boldsymbol{a}^{\boldsymbol{k}^{\prime}}\left(\frac{\tau}{1-\tau}\right)^{|\boldsymbol{k}|}\right) \\
& =\exp \left(\sum_{\mathbf{0} \leq \boldsymbol{k}^{\prime} \leq \boldsymbol{k}} \sum_{\boldsymbol{a} \in A} \beta_{\boldsymbol{a}}^{\boldsymbol{l}} \boldsymbol{a}^{\boldsymbol{k}^{\prime}} \theta_{\boldsymbol{k}^{\prime}, \boldsymbol{k}}\left(\left\{c_{\boldsymbol{s}^{\prime}, \boldsymbol{s}} b_{\boldsymbol{s}}^{\prime}\right\}\right)\left(\frac{\tau}{1-\tau}\right)^{|\boldsymbol{k}|}\right) \\
& =\exp \left(\sum_{\boldsymbol{l} \leq \boldsymbol{k}} \theta_{\boldsymbol{l}, \boldsymbol{k}}\left(\left\{c_{\boldsymbol{s}^{\prime}, \boldsymbol{s}} b_{\boldsymbol{s}}^{\prime}\right\}\right)\left(\frac{\tau}{1-\tau}\right)^{|\boldsymbol{k}|}\right) .
\end{aligned}
$$

Hence

$$
\begin{aligned}
\varphi_{\tau}(\alpha, z) & =b_{\mathbf{0}}^{-m} \prod_{\boldsymbol{l} \in L}\left(\exp \left(\sum_{\boldsymbol{l} \leq \boldsymbol{k}} \theta_{\boldsymbol{l}, \boldsymbol{k}}\left(\left\{c_{\boldsymbol{s}^{\prime}, \boldsymbol{s}} b_{\boldsymbol{s}}^{\prime}\right\}\right)\left(\frac{\tau}{1-\tau}\right)^{|\boldsymbol{k}|}\right)\right)^{\alpha_{\boldsymbol{l}} / \tau^{|\boldsymbol{l}|}} \\
& =b_{\mathbf{0}}^{-m} \prod_{\boldsymbol{l} \in L} \exp \left(\frac{\alpha_{\boldsymbol{l}}}{\tau^{|\boldsymbol{l}|}} \sum_{\boldsymbol{l} \leq \boldsymbol{k}} \theta_{\boldsymbol{l}, \boldsymbol{k}}\left(\left\{c_{\boldsymbol{s}^{\prime}, \boldsymbol{s}} b_{\boldsymbol{s}}^{\prime}\right\}\right)\left(\frac{\tau}{1-\tau}\right)^{|\boldsymbol{k}|}\right) .
\end{aligned}
$$

Hence as $\tau \rightarrow 0$

$$
\begin{aligned}
\varphi_{\tau}(\alpha, z) & \rightarrow b_{\mathbf{0}}^{-m} \prod_{\boldsymbol{l} \in L} \exp \left(\alpha_{\boldsymbol{l}} \theta_{\boldsymbol{l}, \boldsymbol{l}}\left(\left\{c_{\boldsymbol{s}^{\prime}, \boldsymbol{s}} b_{\boldsymbol{s}}^{\prime}\right\}\right)\right) \\
& =b_{\mathbf{0}}^{-m} \prod_{\boldsymbol{l} \in L} \exp \left(\alpha_{\boldsymbol{l}} \theta_{\boldsymbol{l}}\left(\left\{b_{\boldsymbol{s}}^{\prime}\right\}\right)\right),
\end{aligned}
$$

where the last equation follows from Lemma 5.7. Therefore

$$
\varphi_{\tau}(\alpha, z) \rightarrow b_{\mathbf{0}}^{-m} \exp \left(\sum_{\boldsymbol{l} \in L} \alpha_{\boldsymbol{l}} \theta_{\boldsymbol{l}}\left(b^{\prime}\right)\right) \quad(\tau \rightarrow 0) .
$$

Lemma 5.7. Let

$$
\sum_{\mathbf{0} \leq \boldsymbol{k}^{\prime} \leq \boldsymbol{k}} c_{\boldsymbol{k}^{\prime}, \boldsymbol{k}} b_{\boldsymbol{k}}^{\prime} T^{\boldsymbol{k}^{\prime}} S^{\boldsymbol{k}}=\exp \left(\sum_{\mathbf{0} \leq \boldsymbol{k}^{\prime}, \boldsymbol{k}} \theta_{\boldsymbol{k}^{\prime}, \boldsymbol{k}}\left(\left\{c_{\boldsymbol{s}^{\prime}, \boldsymbol{s}} b_{\boldsymbol{s}}^{\prime}\right\}\right) T^{\boldsymbol{k}^{\prime}} S^{\boldsymbol{k}}\right),
$$

where $c_{\mathbf{0}, \mathbf{0}} b_{\mathbf{0}}^{\prime}=1$. Then $\theta_{\boldsymbol{k}^{\prime}, \boldsymbol{k}}=0$ unless $\boldsymbol{k}^{\prime} \leq \boldsymbol{k}$. Moreover, if $c_{\boldsymbol{k}, \boldsymbol{k}}=1$ for all $\boldsymbol{k}$, then

$$
\theta_{\boldsymbol{k}, \boldsymbol{k}}\left(\left\{c_{\boldsymbol{s}^{\prime}, \boldsymbol{s}} b_{\boldsymbol{s}}^{\prime}\right\}\right)=\theta_{\boldsymbol{k}}\left(\left\{b_{\boldsymbol{s}}^{\prime}\right\}\right) .
$$

Proof. The first statement is clear from the Taylor expansion of $\log (1+$ $U)$ :

$$
\log (1+U)=\sum_{n=1}^{\infty} \frac{(-1)^{n-1}}{n} U^{n}
$$


We have

$$
\begin{aligned}
\log \left(\sum_{\mathbf{0} \leq \boldsymbol{k}^{\prime} \leq \boldsymbol{k}} c_{\boldsymbol{k}^{\prime}, \boldsymbol{k}} b_{\boldsymbol{k}}^{\prime} T^{\boldsymbol{k}^{\prime}} S^{\boldsymbol{k}}\right) & =\sum_{n=1}^{\infty} \frac{(-1)^{n-1}}{n}\left(\sum_{\mathbf{0} \leq \boldsymbol{k}^{\prime} \leq \boldsymbol{k}} c_{\boldsymbol{k}^{\prime}, \boldsymbol{k}} b_{\boldsymbol{k}}^{\prime} T^{\boldsymbol{k}^{\prime}} S^{\boldsymbol{k}}\right)^{n} \\
& =\sum_{\mathbf{0} \leq \boldsymbol{k}^{\prime}, \boldsymbol{k}} \theta_{\boldsymbol{k}^{\prime}, \boldsymbol{k}}\left(\left\{c_{\boldsymbol{s}^{\prime}, \boldsymbol{s}} b_{\boldsymbol{s}}^{\prime}\right\}\right) T^{\boldsymbol{k}^{\prime}} S^{\boldsymbol{k}} .
\end{aligned}
$$

Put $U:=T S$. Then

$$
\begin{aligned}
\log \left(\sum_{\mathbf{0} \leq \boldsymbol{k}^{\prime} \leq \boldsymbol{k}} c_{\boldsymbol{k}^{\prime}, \boldsymbol{k}} b_{\boldsymbol{k}}^{\prime} U^{\boldsymbol{k}^{\prime}} S^{\boldsymbol{k}-\boldsymbol{k}^{\prime}}\right) & =\sum_{n=1}^{\infty} \frac{(-1)^{n-1}}{n}\left(\sum_{\mathbf{0} \leq \boldsymbol{k}^{\prime} \leq \boldsymbol{k}} c_{\boldsymbol{k}^{\prime}, \boldsymbol{k}} b_{\boldsymbol{k}}^{\prime} U^{\boldsymbol{k}^{\prime}} S^{\boldsymbol{k}-\boldsymbol{k}^{\prime}}\right)^{n} \\
& =\sum_{\mathbf{0} \leq \boldsymbol{k}^{\prime}, \boldsymbol{k}} \theta_{\boldsymbol{k}^{\prime}, \boldsymbol{k}}\left(\left\{c_{\boldsymbol{s}^{\prime}, \boldsymbol{s}} b_{\boldsymbol{s}}^{\prime}\right\}\right) U^{\boldsymbol{k}^{\prime}} S^{\boldsymbol{k}-\boldsymbol{k}^{\prime}} .
\end{aligned}
$$

Let $S=\mathbf{0}$. Then

$$
\begin{aligned}
\log \left(\sum_{\mathbf{0} \leq \boldsymbol{k}^{\prime}} c_{\boldsymbol{k}^{\prime}, \boldsymbol{k}^{\prime}} b_{\boldsymbol{k}^{\prime}}^{\prime} U^{\boldsymbol{k}^{\prime}}\right) & =\sum_{n=1}^{\infty} \frac{(-1)^{n-1}}{n}\left(\sum_{\mathbf{0} \leq \boldsymbol{k}^{\prime}} c_{\boldsymbol{k}^{\prime}, \boldsymbol{k}^{\prime}} b_{\boldsymbol{k}^{\prime}}^{\prime} U^{\boldsymbol{k}^{\prime}}\right)^{n} \\
& =\sum_{\mathbf{0} \leq \boldsymbol{k}^{\prime}} \theta_{\boldsymbol{k}^{\prime}, \boldsymbol{k}^{\prime}}\left(\left\{c_{\boldsymbol{s}^{\prime}, \boldsymbol{s}} b_{\boldsymbol{s}}^{\prime}\right\}\right) U^{\boldsymbol{k}^{\prime}}
\end{aligned}
$$

Since $c_{\boldsymbol{k}^{\prime}, \boldsymbol{k}^{\prime}}=1$ for all $\boldsymbol{k}^{\prime}$,

$$
\begin{aligned}
\log \left(\sum_{\mathbf{0} \leq \boldsymbol{k}^{\prime}} b_{\boldsymbol{k}^{\prime}}^{\prime} U^{\boldsymbol{k}^{\prime}}\right) & =\sum_{n=1}^{\infty} \frac{(-1)^{n-1}}{n}\left(\sum_{\mathbf{0} \leq \boldsymbol{k}^{\prime}} b_{\boldsymbol{k}^{\prime}}^{\prime} U^{\boldsymbol{k}^{\prime}}\right)^{n} \\
& =\sum_{\mathbf{0} \leq \boldsymbol{k}^{\prime}} \theta_{\boldsymbol{k}^{\prime}, \boldsymbol{k}^{\prime}}\left(\left\{c_{\boldsymbol{s}^{\prime}, \boldsymbol{s}} b_{\boldsymbol{s}}^{\prime}\right\}\right) U^{\boldsymbol{k}^{\prime}}
\end{aligned}
$$

which means

$$
\theta_{\boldsymbol{k}^{\prime}, \boldsymbol{k}^{\prime}}\left(\left\{c_{\boldsymbol{s}^{\prime}, \boldsymbol{s}} b_{\boldsymbol{s}}^{\prime}\right\}\right)=\theta_{\boldsymbol{k}^{\prime}}\left(\left\{b_{\boldsymbol{s}}^{\prime}\right\}\right) \quad\left(\forall \boldsymbol{k}^{\prime}\right)
$$

\section{THE NORMALIZER OF $\mathfrak{a}$ AND ITS ACTION}

In this section, we consider the normalizer $N_{G}(\mathfrak{a})$ of $\mathfrak{a}$ and its action following [8]. Using the results in this section, we specialize parameters of hypergeometric systems associated with principal nilpotent $p$-tuples in the next section.

Put $L_{+}:=L \backslash\{\boldsymbol{0}\}$ and $\mathfrak{a}_{+}:=\left\langle e^{\boldsymbol{l}} \mid \boldsymbol{l} \in L_{+}\right\rangle$. Note that $N_{G}(\exp \mathfrak{a})=$ $N_{G}\left(\exp \mathfrak{a}_{+}\right) \supseteq N_{G}\left(\mathfrak{a}_{+}\right)=N_{G}(\mathfrak{a})$. The action of $N_{G}(\mathfrak{a})$ on $Z / G L(m)$ induces that of $N_{G}(\mathfrak{a})$ on $\exp (\mathfrak{a}) \backslash Z / G L(m)$. 
Let $\chi_{\alpha}$ denote the (multivalued) character of exp $\mathfrak{a}$ whose differential is equal to $\alpha=\sum_{\boldsymbol{l}} \alpha_{l}\left(e^{\boldsymbol{l}}\right)^{*} \in \mathfrak{a}^{*}$. Then we have the following commutative diagram:

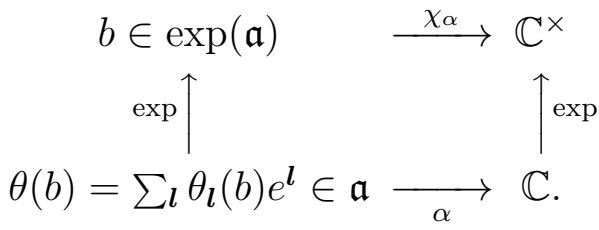

Namely we have

$$
\chi_{\alpha}(b)=\exp (\alpha(\theta(b))) .
$$

Lemma 6.1. $\theta\left(g b g^{-1}\right)=\operatorname{Ad}(g)(\theta(b))$ for $g \in N_{G}(\mathfrak{a}), b \in \exp \mathfrak{a}$.

Proof. We have

$$
g b g^{-1}=g \exp (\theta(b)) g^{-1}=\exp \left(g \theta(b) g^{-1}\right)=\exp (\operatorname{Ad}(g)(\theta(b))) .
$$

Hence $\theta\left(g b g^{-1}\right)=\operatorname{Ad}(g)(\theta(b))$.

Lemma 6.2. $\chi_{\alpha}\left(g b g^{-1}\right)=\chi_{\operatorname{Ad}^{*}(g) \alpha}(b)$ for $g \in N_{G}(\mathfrak{a}), b \in \exp \mathfrak{a}$, where $\mathrm{Ad}^{*}$ is the coadjoint action.

Proof. By Lemma 6.1,

$$
\begin{aligned}
\chi_{\alpha}\left(g b g^{-1}\right) & =\exp \left(\alpha\left(\theta\left(g b g^{-1}\right)\right)\right)=\exp (\alpha(\operatorname{Ad}(g)(\theta(b)))) \\
& =\exp \left(\left(\operatorname{Ad}^{*}(g)(\alpha)\right)(\theta(b))\right)=\chi_{\operatorname{Ad}^{*}(g) \alpha}(b) .
\end{aligned}
$$

Lemma 6.3. Let $b=\sum_{\boldsymbol{l}} b_{\boldsymbol{l}} e^{\boldsymbol{l}} \in \exp \mathfrak{a}$. Then

$$
b=b_{\mathbf{0}} \exp \left(\sum_{\boldsymbol{k} \ngtr \mathbf{0}} \theta_{\boldsymbol{k}}\left(b^{\prime}\right) e^{\boldsymbol{k}}\right),
$$

where $b_{\boldsymbol{l}}^{\prime}=b_{\boldsymbol{l}} / b_{\mathbf{0}}$. Hence

$$
\chi_{\alpha}(b)=b_{\mathbf{0}}^{\alpha_{0}} \exp \left(\sum_{\boldsymbol{k} \ngtr \mathbf{0}} \alpha_{\boldsymbol{k}} \theta_{\boldsymbol{k}}\left(b^{\prime}\right)\right) .
$$

Proof. This is clear, since $b=b_{\mathbf{0}} \sum_{\boldsymbol{l}} b_{\boldsymbol{l}}^{\prime} e^{\boldsymbol{l}}$.

We denote by $\iota$ the isomorphism $\mathfrak{a} \ni \sum_{\boldsymbol{l}} c_{\boldsymbol{l}} e^{\boldsymbol{l}} \mapsto\left[c_{\boldsymbol{l}}\right]_{\boldsymbol{l}} \in \mathbb{C}^{L}$, and by $\iota^{*}$ its dual isomorphism: $\mathbb{C}^{L} \rightarrow \mathfrak{a}^{*}$. For $g \in N_{G}(\mathfrak{a})$, let $M(g)$ denote the matrix representation of $\operatorname{Ad}(g)$ on $\mathfrak{a}$ with respect to the basis $\left\{e^{\boldsymbol{l}} \mid \boldsymbol{l} \in L\right\}$.

Then by the definitions

$$
\operatorname{Ad}(g)\left(\iota^{-1}(h)\right)=\iota^{-1}(M(g) h), \quad \operatorname{Ad}^{*}(g)\left(\iota^{*}(h)\right)=\iota^{*}\left({ }^{t} M(g) h\right)
$$

for $h \in \mathbb{C}^{L}$.

Proposition 6.4. $\phi(\alpha, M(g) z, t)=\phi\left(\operatorname{Ad}^{*}(g) \alpha, z, t\right)$ for $g \in N_{G}(\mathfrak{a})$. 
Proof. Recall the definition of $\phi(\alpha, z, t)$ in Proposition 4.3;

$$
\phi(\alpha, z, t)=\chi_{\alpha}\left(\sum_{l} b_{\boldsymbol{l}} e^{\boldsymbol{l}}\right)=\chi_{\alpha}\left(\iota^{-1}\left(\left[b_{\boldsymbol{l}}\right]_{\boldsymbol{l}}\right)\right)
$$

by Lemma 6.3 , where $b_{\boldsymbol{l}}=(z t)_{\boldsymbol{l}}$. Hence by (18) we have

$$
\begin{aligned}
\phi(\alpha, M(g) z, t) & =\chi_{\alpha}\left(\iota^{-1}\left(\left[(M(g) z t)_{l}\right]_{l}\right)\right)=\chi_{\alpha}\left(\operatorname{Ad}(g) \iota^{-1}\left(\left[(z t)_{l}\right]_{l}\right)\right) \\
& =\chi_{\operatorname{Ad}^{*}(g) \alpha}\left(\iota^{-1}\left(\left[(z t)_{l}\right]_{l}\right)\right)=\phi\left(\operatorname{Ad}^{*}(g) \alpha, z, t\right) .
\end{aligned}
$$

Corollary 6.5. $\psi(\alpha, M(g) z)=\psi\left(\operatorname{Ad}^{*}(g) \alpha, z\right)$ for $g \in N_{G}(\mathfrak{a})$.

To describe the matrix representation of $\operatorname{Ad}(g)\left(g \in N_{G}(\mathfrak{a})\right)$, we introduce some notation.

Put

$$
\begin{aligned}
& f_{i}(x, T):=\sum_{\boldsymbol{a} \in \mathbb{N}^{p}} x_{\boldsymbol{a}}^{(i)} T^{\boldsymbol{a}} \quad(i=1,2, \ldots, p) \\
& f(x, T)^{\boldsymbol{l}}:=\prod_{i=1}^{p}\left(f_{i}(x, T)\right)^{l_{i}}=\sum_{\boldsymbol{l}^{\prime} \in \mathbb{N}^{p}} \phi_{\boldsymbol{l}^{\prime}, \boldsymbol{l}}(x) T^{\boldsymbol{l}^{\prime}}
\end{aligned}
$$

for $\boldsymbol{l} \in \mathbb{N}^{p}$.

Lemma 6.6. (1) $\phi_{\boldsymbol{l}^{\prime}, \mathbf{0}}(x)= \begin{cases}1 & \left(\boldsymbol{l}^{\prime}=\mathbf{0}\right) \\ 0 & \text { (otherwise) } .\end{cases}$

(2) $\phi_{\boldsymbol{l}^{\prime}, \boldsymbol{l}}(x)=\sum_{\sum_{i=1}^{p} \sum_{j=1}^{l_{i}} \boldsymbol{a}_{i j}=\boldsymbol{l}^{\prime}} \prod_{i=1}^{p} x_{\boldsymbol{a}_{i 1}}^{(i)} \cdots x_{\boldsymbol{a}_{i l_{i}}}^{(i)}$.

(3) $\phi_{\boldsymbol{l}^{\prime}, \boldsymbol{l}}(x)=0$ for $|\boldsymbol{l}|>\left|\boldsymbol{l}^{\prime}\right|$.

(4) $\phi_{\boldsymbol{k}, \boldsymbol{l}+\boldsymbol{l}^{\prime}}(x)=\sum_{\boldsymbol{k}_{1}+\boldsymbol{k}_{2}=\boldsymbol{k}} \phi_{\boldsymbol{k}_{1}, l}(x) \phi_{\boldsymbol{k}_{2}, \boldsymbol{l}^{\prime}}(x)$.

Proof. (1) follows from $f(x, T)^{\mathbf{0}}=1$.

Since we have

$$
\begin{aligned}
f(x, T)^{\boldsymbol{l}} & =\prod_{i=1}^{p}\left(\sum_{\boldsymbol{a} \in \mathbb{N} p} x_{\boldsymbol{a}}^{(i)} T^{\boldsymbol{a}}\right)^{l_{i}} \\
& =\prod_{i=1}^{p}\left(\sum_{\boldsymbol{a}_{i 1}, \ldots, \boldsymbol{a}_{i l_{i}} \in \mathbb{N}^{p}} x_{\boldsymbol{a}_{i 1}}^{(i)} \cdots x_{\boldsymbol{a}_{i l_{i}}^{(i)}} T^{\sum_{j=1}^{l_{i}} \boldsymbol{a}_{i j}}\right)
\end{aligned}
$$

we obtain (2).

Since $\left|\sum_{i=1}^{p} \sum_{j=1}^{l_{i}} \boldsymbol{a}_{i j}\right| \geq|\boldsymbol{l}|$, (3) follows from (2).

The equation $f(x, T)^{\boldsymbol{l}+\boldsymbol{l}^{\prime}}=f(x, T)^{\boldsymbol{l}} f(x, T)^{\boldsymbol{l}^{\prime}}$ leads to (4).

Corollary 6.7. Suppose that $|\boldsymbol{l}|=\left|\boldsymbol{l}^{\prime}\right|$. Then any term of $\phi_{\boldsymbol{l}^{\prime}, \boldsymbol{l}}$ is a product of $x_{1_{j}}^{(i)}(i, j=1,2, \ldots, p)$.

Proof. This is clear from Lemma 6.6 (2). 
Corollary 6.8. Let $L_{k}$ be a subset of $\mathbb{N}_{k}^{p}:=\left\{\boldsymbol{l} \in \mathbb{N}^{p}|| \boldsymbol{l} \mid=k\right\}(k>0)$. Suppose that $C=\left[c_{1_{j}}^{(i)}\right]_{1 \leq i, j \leq p} \in G L(p)$ satisfies $\phi_{\boldsymbol{l}^{\prime}, \boldsymbol{l}}(C)=0$ for all $\boldsymbol{l}^{\prime} \in L_{k}, \boldsymbol{l} \in \mathbb{N}_{k}^{p} \backslash L_{k}$.

Then $\operatorname{det}\left(\left[\phi_{\boldsymbol{l}^{\prime}, \boldsymbol{l}}\right]_{\boldsymbol{l}^{\prime}, \boldsymbol{l} \in L_{k}}\right) \neq 0$.

Proof. First note that for $\boldsymbol{l}^{\prime}, \boldsymbol{l} \in \mathbb{N}_{k}^{p}$ the notation $\phi_{\boldsymbol{l}^{\prime}, \boldsymbol{l}}(C)$ is justified by Corollary 6.7.

Let $U=\oplus_{i=1}^{p} \mathbb{C} \boldsymbol{u}_{i}$, and let $S_{k}(U)$ be the space of symmetric product of degree $k$. Then there exists a natural representation

$$
\rho_{k}: G L(U) \rightarrow G L\left(S_{k}(U)\right) .
$$

With respect to the basis $\left\{\boldsymbol{u}^{\boldsymbol{l}} \mid \boldsymbol{l} \in \mathbb{N}_{k}^{p}\right\}, \rho_{k}(C)$ is represented by $\left[\phi_{\boldsymbol{l}^{\prime}, \boldsymbol{l}}(C)\right]_{\boldsymbol{l}^{\prime}, \boldsymbol{l} \in \mathbb{N}_{k}^{p}}$. Hence

$$
\begin{aligned}
0 & \neq \operatorname{det}\left(\left[\phi_{\boldsymbol{l}^{\prime}, \boldsymbol{l}}(C)\right]_{\boldsymbol{l}^{\prime}, \boldsymbol{l} \in \mathbb{N}_{k}^{p}}\right) \\
& =\operatorname{det}\left(\left[\phi_{\boldsymbol{l}^{\prime}, \boldsymbol{l}}(C)\right]_{\boldsymbol{l}^{\prime}, \boldsymbol{l} \in L_{k}}\right) \operatorname{det}\left(\left[\phi_{\boldsymbol{l}^{\prime}, \boldsymbol{l}}(C)\right]_{\boldsymbol{l}^{\prime}, \boldsymbol{l} \in \mathbb{N}_{k}^{p} \backslash L_{k}}\right)
\end{aligned}
$$

by the assumption. Hence we have $\operatorname{det}\left(\left[\phi_{\boldsymbol{l}^{\prime}, \boldsymbol{l}}\right]_{\boldsymbol{l}^{\prime}, \boldsymbol{l} \in L_{k}}\right) \neq 0$.

Proposition 6.9. Let $g \in N_{G}(\mathfrak{a})$, and let

$$
\operatorname{Ad}(g) e_{i}=\sum_{\boldsymbol{l} \in L_{+}} c_{\boldsymbol{l}}^{(i)} e^{\boldsymbol{l}}=f_{i}\left(\left\{c_{\boldsymbol{l}}^{(i)}\right\}, e\right)
$$

Then

$$
\operatorname{Ad}(g) e^{\boldsymbol{l}}=\sum_{\boldsymbol{l}^{\prime} \in L_{+}} \phi_{\boldsymbol{l}^{\prime}, \boldsymbol{l}}\left(\left\{c_{\boldsymbol{k}}^{(i)}\right\}\right) e^{\boldsymbol{l}^{\prime}}=f\left(\left\{c_{\boldsymbol{k}}^{(i)}\right\}, e\right)^{\boldsymbol{l}} .
$$

Proof. This is immediate from $\operatorname{Ad}(g) e^{l}=\prod_{i=1}^{p}\left(\operatorname{Ad}(g) e_{i}\right)^{l_{i}}$.

For $g \in N_{G}(\mathfrak{a})$, let $M(g)$ denote the matrix representation of $\operatorname{Ad}(g)$ on $\mathfrak{a}$ with respect to the basis $\left\{e^{\boldsymbol{l}} \mid \boldsymbol{l} \in L\right\}$.

Corollary 6.10. Put $\phi_{\mathbf{0}, l}=\delta_{\mathbf{0}, \boldsymbol{l}}$. Then

$$
M(g)=\left[m(g)_{\boldsymbol{l}^{\prime}, l}\right]_{l, l^{\prime} \in L}=\left[\phi_{\boldsymbol{l}^{\prime}, \boldsymbol{l}}\left(\left\{c_{\boldsymbol{k}}^{(i)}\right\}\right)\right]_{\boldsymbol{l}, \boldsymbol{l}^{\prime} \in L},
$$

where $c_{l}^{(i)}$ are those in Proposition 6.9.

Proof. Since $\operatorname{Ad}(g) e^{\boldsymbol{l}}$ is nilpotent, we have $m(g)_{\mathbf{0}, \boldsymbol{l}}=\delta_{\mathbf{0}, \boldsymbol{l}}$.

Definition 6.11. (1) $\boldsymbol{a} \in L$ is called an inner corner of $L$, if $\boldsymbol{a}+$ $1_{i} \notin L$ for any $i=1,2, \ldots, p$.

(2) $\boldsymbol{a} \in \mathbb{N}^{p}$ is called an outer corner of $L$, if $\boldsymbol{a} \notin L$, and for every $i=1,2, \ldots, p$ we have $\boldsymbol{a}-1_{i} \notin \mathbb{N}^{p}$ or $\boldsymbol{a}-1_{i} \in L$. 
Example 6.12. Let $p=2$. Then $L$ can be given by a Young diagram (see Remark 3.4). Suppose that $L$ corresponds to the Young diagram $(5,5,3,2,1)$.

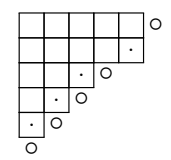

Then in (19) • indicates an inner corner, and o an outer corner.

Lemma 6.13. Let $g \in N_{G}(\mathfrak{a})$, and let $\operatorname{Ad}(g) e_{i}=\sum_{\boldsymbol{l} \in L_{+}} c_{\boldsymbol{l}}^{(i)} e^{\boldsymbol{l}}$.

(1) Let $\boldsymbol{a}$ be an inner corner. Then $\prod_{i=1}^{p}\left(\sum_{\boldsymbol{l} \in L_{+}} c_{\boldsymbol{l}}^{(i)} e^{\boldsymbol{l}}\right)^{a_{i}} \neq 0$.

(2) Let $\boldsymbol{a}$ be an outer corner. Then $\prod_{i=1}^{p}\left(\sum_{\boldsymbol{l} \in L_{+}} c_{\boldsymbol{l}}^{(i)} e^{\boldsymbol{l}}\right)^{a_{i}}=0$.

Proof. Note that

$$
f(c, e)^{\boldsymbol{a}}=\operatorname{Ad}(g) e^{\boldsymbol{a}}=\prod_{i=1}^{p}\left(\sum_{\boldsymbol{l} \in L_{+}} c_{\boldsymbol{l}}^{(i)} e^{\boldsymbol{l}}\right)^{a_{i}} .
$$

Hence the assertions are immediate, since an inner corner belongs to $L$ and an outer corner does not.

Remark 6.14. Let $g \in N_{G}(\mathfrak{a})$, and let $\operatorname{Ad}(g) e_{i}=\sum_{\boldsymbol{l} \in L_{+}} c_{\boldsymbol{l}}^{(i)} e^{\boldsymbol{l}}$. Suppose that $\boldsymbol{l}$ is an outer corner of $L$. Then, by Lemma 6.6 (4),

$$
\begin{aligned}
0 & =\phi_{\boldsymbol{k}, \boldsymbol{l}}(c)=\sum_{\boldsymbol{k}_{1}+\cdots+\boldsymbol{k}_{p}=\boldsymbol{k}} \prod_{i=1}^{p} \phi_{\boldsymbol{k}_{i}, l_{i} 1_{i}}(c) \\
& =\sum_{\sum_{i=1}^{p} \sum_{j=1}^{l_{i}} \boldsymbol{k}_{i j}=\boldsymbol{k}} \prod_{i=1}^{p} \prod_{j=1}^{l_{i}} \phi_{\boldsymbol{k}_{i j}, 1_{i}}(c) \\
& =\sum_{\sum_{i=1}^{p} \sum_{j=1}^{l_{i}} \boldsymbol{k}_{i j}=\boldsymbol{k}} \prod_{i=1}^{p} \prod_{j=1}^{l_{i}} c_{\boldsymbol{k}_{i j}}^{(i)}
\end{aligned}
$$

for $\boldsymbol{k} \in L_{+}$. These equations are equivalent to Lemma 6.13.

Example 6.15. Let $p=1$, and $L=\{0,1, \ldots, n-1\}$. Then $n-1$ is the inner corner and $n$ is the outer corner. We have

$$
\left(\sum_{l=1}^{n-1} c_{l} e^{l}\right)^{n-1} \neq 0, \quad\left(\sum_{l=1}^{n-1} c_{l} e^{l}\right)^{n}=0 .
$$

We obtain $c_{1} \neq 0$ from the first equation.

Example 6.16. Let $p=2$, and $L=\{(i, 0) \mid 0 \leq i \leq n-2\} \cup\{(1,0)\}$. Then the inner corners are $(n-2,0)$ and $(0,1)$. The outer corners are $(n-1,0),(1,1)$, and $(0,2)$. 
Then we have

$$
\begin{aligned}
& \left(\sum_{l=1}^{n-2} c_{(l, 0)}^{(1)} e_{1}^{l}+c_{(0,1)}^{(1)} e_{2}\right)^{n-2} \neq 0 \\
& \left(\sum_{l=1}^{n-2} c_{(l, 0)}^{(2)} e_{1}^{l}+c_{(0,1)}^{(2)} e_{2}\right) \neq 0 \\
& \left(\sum_{l=1}^{n-2} c_{(l, 0)}^{(1)} e_{1}^{l}+c_{(0,1)}^{(1)} e_{2}\right)^{n-1}=0 \\
& \left(\sum_{l=1}^{n-2} c_{(l, 0)}^{(1)} e_{1}^{l}+c_{(0,1)}^{(1)} e_{2}\right)\left(\sum_{l=1}^{n-2} c_{(l, 0)}^{(2)} e_{1}^{l}+c_{(0,1)}^{(2)} e_{2}\right)=0 \\
& \left(\sum_{l=1}^{n-2} c_{(l, 0)}^{(2)} e_{1}^{l}+c_{(0,1)}^{(2)} e_{2}\right)^{2}=0 .
\end{aligned}
$$

From these equations, we obtain $c_{(1,0)}^{(1)} \neq 0, c_{(l, 0)}^{(2)}=0$ for $l \leq n-3$.

Put

$$
L_{k}:=\left\{\boldsymbol{l} \in L_{+}|| \boldsymbol{l} \mid=k\right\} .
$$

Lemma 6.17. Let $g \in N_{G}(\mathfrak{a})$, and let $\operatorname{Ad}(g) e_{i}=\sum_{\boldsymbol{l} \in L_{+}} c_{\boldsymbol{l}}^{(i)} e^{\boldsymbol{l}}$. For any $k$,

$$
\operatorname{det}\left[\phi_{\boldsymbol{l}^{\prime}, \boldsymbol{l}}\left(\left\{c_{\boldsymbol{k}}^{(i)}\right\}\right)\right]_{\boldsymbol{l}, \boldsymbol{l}^{\prime} \in L_{k}} \neq 0
$$

Proof. Since $\operatorname{Ad}(g) \mathfrak{a}^{k}=\mathfrak{a}^{k}$ for any $k$, we have $\operatorname{Ad}(g)\left(\mathfrak{a}^{k} / \mathfrak{a}^{k+1}\right)=\mathfrak{a}^{k} / \mathfrak{a}^{k+1}$. Hence the assertion holds.

Example 6.18. Let $p=2$, and $L=\{(i, 0) \mid 0 \leq i \leq n-2\} \cup\{(1,0)\}$. Then $L_{1}=\{(1,0),(0,1)\}$. Hence

$$
\operatorname{det}\left[\begin{array}{ll}
c_{(1,0)}^{(1)} & c_{(1,0)}^{(2)} \\
c_{(0,1)}^{(1)} & c_{(0,1)}^{(2)}
\end{array}\right] \neq 0 .
$$

For $2 \leq k \leq n-2, L_{k}=\{(k, 0)\}$. Hence

$$
0 \neq \phi_{(k, 0),(k, 0)}(c)=\phi_{(1,0),(1,0)}(c)^{k}=\left(c_{(1,0)}^{(1)}\right)^{k} \text {. }
$$

Hence $c_{(1,0)}^{(1)} \neq 0$, which is the inner corner condition.

Proposition 6.19. Let $g \in N_{G}(\mathfrak{a})$, and let $\operatorname{Ad}(g) e_{i}=\sum_{\boldsymbol{l} \in L_{+}} c_{\boldsymbol{l}}^{(i)} e^{\boldsymbol{l}}$. Suppose that $A=\alpha(0)+L$. Put $\boldsymbol{u}_{\boldsymbol{l}}:=v_{\alpha(0)+\boldsymbol{l}}=e^{\boldsymbol{l}} v_{\alpha(0)}$. Define $\tilde{g} \in G L(V)$ by

$$
\tilde{g} \boldsymbol{u}_{\boldsymbol{l}}=\sum_{\boldsymbol{l}^{\prime}} \phi_{\boldsymbol{l}^{\prime}, \boldsymbol{l}}(c) \boldsymbol{u}_{\boldsymbol{l}^{\prime}}
$$

Then $\tilde{g} \in N_{G}(\mathfrak{a})$ and $M(\tilde{g})=\tilde{g}=M(g)$. 
Proof. We know that $M(g)=\left[\phi_{\boldsymbol{l}^{\prime}, \boldsymbol{l}}(c)\right]$ is invertible. Let $\left[\psi_{\boldsymbol{l}^{\prime}, l}\right]$ be its inverse. Then

$$
\tilde{g}^{-1} \boldsymbol{u}_{\boldsymbol{l}}=\sum_{\boldsymbol{l}^{\prime}} \psi_{\boldsymbol{l}^{\prime}, \boldsymbol{l}} \boldsymbol{u}_{\boldsymbol{l}^{\prime}}
$$

Hence by Lemma $6.6(4)$

$$
\begin{aligned}
\tilde{g} e^{\boldsymbol{k}} \tilde{g}^{-1} \boldsymbol{u}_{\boldsymbol{l}} & =\sum_{\boldsymbol{l}^{\prime}} \psi_{\boldsymbol{l}^{\prime}, l} \tilde{g} \boldsymbol{u}_{\boldsymbol{l}^{\prime}+\boldsymbol{k}} \\
& =\sum_{\boldsymbol{l}^{\prime}, \boldsymbol{l}^{\prime \prime}} \psi_{\boldsymbol{l}^{\prime}, \boldsymbol{l}} \phi_{\boldsymbol{l}^{\prime \prime}, \boldsymbol{l}^{\prime}+\boldsymbol{k}} \boldsymbol{u}_{\boldsymbol{l}^{\prime \prime}} \\
& =\sum_{\boldsymbol{l}^{\prime}, \boldsymbol{l}_{1}, \boldsymbol{l}_{2}} \psi_{\boldsymbol{l}^{\prime}, \boldsymbol{l}} \phi_{\boldsymbol{l}_{1}, \boldsymbol{l}^{\prime}} \phi_{\boldsymbol{l}_{2}, \boldsymbol{k}} \boldsymbol{u}_{\boldsymbol{l}_{1}+\boldsymbol{l}_{2}} \\
& =\sum_{\boldsymbol{l}_{1}, \boldsymbol{l}_{2}} \delta_{\boldsymbol{l}_{1}, \boldsymbol{l}} \phi_{\boldsymbol{l}_{2}, \boldsymbol{k}} \boldsymbol{u}_{\boldsymbol{l}_{1}+\boldsymbol{l}_{2}} \\
& =\sum_{\boldsymbol{l}_{2}} \phi_{\boldsymbol{l}_{2}, \boldsymbol{k}} \boldsymbol{u}_{\boldsymbol{l}+\boldsymbol{l}_{2}}=\sum_{\boldsymbol{l}_{2}} \phi_{\boldsymbol{l}_{2}, \boldsymbol{k}} e^{\boldsymbol{l}_{2}} \boldsymbol{u}_{\boldsymbol{l}}
\end{aligned}
$$

Hence

$$
\tilde{g} e^{\boldsymbol{k}} \tilde{g}^{-1}=\sum_{\boldsymbol{l}_{2}} \phi_{\boldsymbol{l}_{2}, \boldsymbol{k}} e^{\boldsymbol{l}_{2}}
$$

We have thus proved $\tilde{g} \in N_{G}(\mathfrak{a})$ and $M(\tilde{g})=\tilde{g}=M(g)$.

Corollary 6.20. Let $c_{\boldsymbol{l}}^{(i)}\left(1 \leq i \leq p, \boldsymbol{l} \in L_{+}\right)$satisfy the conditions

(1) $\operatorname{det}\left(\left[\phi_{\boldsymbol{l}^{\prime}, \boldsymbol{l}}(c)\right]_{\boldsymbol{l}, \boldsymbol{l}^{\prime} \in L_{+}}\right) \neq 0$.

(2) $\phi_{\boldsymbol{l}^{\prime}, \boldsymbol{l}}(c)=0$ for every $\boldsymbol{l} \notin L_{+}, \boldsymbol{l}^{\prime} \in L_{+}$.

Then $g=\left[\phi_{\boldsymbol{l}^{\prime}, l}(c)\right]_{\boldsymbol{l}, \boldsymbol{l}^{\prime} \in L} \in N_{G}(\mathfrak{a})$ and $M(g)=g$.

Proof. This is clear from the proof of Proposition 6.19.

Lemma 6.21. The following two conditions are equivalent:

(1) $\prod_{i=1}^{p}\left(\sum_{\boldsymbol{l}^{\prime} \in L_{+}} c_{\boldsymbol{l}}^{(i)} e^{\boldsymbol{l}^{\prime}}\right)^{l_{i}}=0$ for all outer corners $\boldsymbol{l}$.

(2) $\phi_{\boldsymbol{l}^{\prime}, \boldsymbol{l}}(c)=0$ for every $\boldsymbol{l} \notin L_{+}, \boldsymbol{l}^{\prime} \in L_{+}$.

Proof. The condition (2) is equivalent to the condition

$$
f(c, e)^{\boldsymbol{l}}=0 \text { for all } \boldsymbol{l} \notin L_{+},
$$

which is equivalent to the condition

$$
f(c, e)^{\boldsymbol{l}}=0 \text { for all outer corners } \boldsymbol{l},
$$

which is exactly the condition (1).

Proposition 6.22. Each element of $N_{G}\left(\mathfrak{a}_{+}\right) / Z_{G}\left(\mathfrak{a}_{+}\right)$is uniquely written as $\left[\phi_{\boldsymbol{l}^{\prime}, \boldsymbol{l}}(c)\right]_{\boldsymbol{l}^{\prime}, \boldsymbol{l} \in L_{+}}$with $c=\left(c_{\boldsymbol{l}}^{(i)}: 1 \leq i \leq p ; \boldsymbol{l} \in L_{+}\right)$satisfying

(1) $\operatorname{det}\left[c_{1_{j}}^{(i)}\right]_{1 \leq i, j \leq p} \neq 0$. 
(2) $f(c, e)^{\boldsymbol{l}}=0$ for all outer corners $\boldsymbol{l}$.

Proof. The condition (1) in Corollary 6.20 is equivalent to the condition

$$
\operatorname{det}\left(\left[\phi_{\boldsymbol{l}^{\prime}, \boldsymbol{l}}(c)\right]_{\boldsymbol{l}, \boldsymbol{l}^{\prime} \in L_{k}}\right) \neq 0 \quad(\forall k>0)
$$

by Lemma 6.6 (3). Then by Corollary 6.8 we see that the conditions

$$
\operatorname{det}\left(\left[\phi_{\boldsymbol{l}^{\prime}, \boldsymbol{l}}(c)\right]_{\boldsymbol{l}, \boldsymbol{l}^{\prime} \in L_{k}}\right) \neq 0 \quad(k>1)
$$

are derived from the other conditions.

\section{Type $(n-1,1)$}

As stated in the paragraph just above Lemma 2.1, when $\mathfrak{a}$ is associated with $L=\{0,1,2, \ldots, n-1\}=\{(l, 0) \mid 0 \leq l \leq n-1\}$, which we may call type $(n)$, the system $M_{\mathfrak{a}, \alpha}$ is the generalized Airy system [5].

In this section, we consider the system $M_{\mathfrak{a}, \alpha}$ of type $(n-1,1)$, which we also denote by $M_{(n-1,1), \alpha}$. Namely let $L=\{(l, 0) \mid 0 \leq l \leq n-2\} \cup$ $\{(0,1)\}$ and $\mathfrak{a}=\left\langle\boldsymbol{e}^{\boldsymbol{l}} \mid \boldsymbol{l} \in L\right\rangle$

$$
L=\square \cdot \square .
$$

Then the system $M_{\mathfrak{a}, \alpha}$ with a parameter vector $\alpha \in \mathfrak{a}_{+}^{*} \simeq \mathbb{C}^{L_{+}}$is $\mathcal{D}_{Z} / J_{\mathfrak{a}, \alpha}$, where the left ideal $J_{\mathfrak{a}, \alpha}$ is generated by the following:

$$
\begin{aligned}
& \frac{\partial}{\partial z_{\boldsymbol{a}, j_{1}}} \frac{\partial}{\partial z_{\boldsymbol{b}, j_{2}}}-\frac{\partial}{\partial z_{\boldsymbol{a}, j_{2}}} \frac{\partial}{\partial z_{\boldsymbol{b}, j_{1}}} \quad\left(\boldsymbol{a}, \boldsymbol{b} \in L ; 1 \leq j_{1}, j_{2} \leq m\right), \\
& \sum_{\boldsymbol{a} \in L} z_{\boldsymbol{a}, j_{1}} \frac{\partial}{\partial z_{\boldsymbol{a}, j_{2}}}+\delta_{j_{1} j_{2}} \quad\left(1 \leq j_{1}, j_{2} \leq m\right), \\
& \sum_{i=0}^{n-k-2} \sum_{j=1}^{m} z_{(i, 0), j} \frac{\partial}{\partial z_{(i+k, 0), j}}+\alpha_{(k, 0)} \quad(k=1, \ldots, n-2), \\
& \sum_{j=1}^{m} z_{(0,0), j} \frac{\partial}{\partial z_{(0,1), j}}+\alpha_{(0,1)} .
\end{aligned}
$$

Note that by (24) we have

$$
\sum_{i=0}^{n-2} \sum_{j=1}^{m} z_{(i, 0), j} \frac{\partial}{\partial z_{(i, 0), j}}+\sum_{j=1}^{m} z_{(0,1), j} \frac{\partial}{\partial z_{(0,1), j}}+m=0
$$

and thus $\alpha_{(0,0)}$ is always equal to $m$; hence we consider $\alpha \in \mathfrak{a}_{+}^{*} \simeq \mathbb{C}^{L_{+}}$.

In this section, we prove that $M_{(n-1,1), \alpha}$ is generically holonomic (Theorem 7.2), and is reduced to $M_{(n-1), \beta}$, when $\alpha_{(n-2,0)} \neq 0$ (Proposition 7.5, Theorem 7.6). 
Lemma 7.1. Let $\alpha=\left(\alpha_{\boldsymbol{l}}\right)_{\boldsymbol{l} \in L_{+}} \in \mathbb{C}^{L_{+}}$be a parameter vector. Then

$$
\alpha_{(n-2,0)} \frac{\partial}{\partial z_{(0,1), j}}-\alpha_{(0,1)} \frac{\partial}{\partial z_{(n-2,0), j}} \in J_{\mathfrak{a},-\alpha}
$$

for all $j=1, \ldots, m$.

Proof. Since

$$
\sum_{l=1}^{m} z_{(0,0), l} \frac{\partial}{\partial z_{(n-2,0), l}}-\alpha_{(n-2,0)}, \sum_{l=1}^{m} z_{(0,0), l} \frac{\partial}{\partial z_{(0,1), l}}-\alpha_{(0,1)}
$$

belong to the ideal $J_{\mathfrak{a},-\alpha}$, we see by $(23)$ that

$$
\begin{aligned}
\alpha_{(n-2,0)} & \frac{\partial}{\partial z_{(0,1), j}}-\alpha_{(0,1)} \frac{\partial}{\partial z_{(n-2,0), j}} \\
\equiv & \frac{\partial}{\partial z_{(0,1), j}}\left(\sum_{l=1}^{m} z_{(0,0), l} \frac{\partial}{\partial z_{(n-2,0), l}}-\alpha_{(n-2,0)}\right) \\
& -\frac{\partial}{\partial z_{(n-2,0), j}}\left(\sum_{l=1}^{m} z_{(0,0), l} \frac{\partial}{\partial z_{(0,1), l}}-\alpha_{(0,1)}\right)
\end{aligned}
$$

belongs to $J_{\mathfrak{a},-\alpha}$.

Theorem 7.2. Let $\alpha=\left(\alpha_{\boldsymbol{l}}\right)_{\boldsymbol{l} \in L_{+}} \in \mathbb{C}^{L_{+}}$satisfy $\alpha_{(n-2,0)} \neq 0$. Then $M_{\mathfrak{a},-\alpha}$ is generically holonomic.

Proof. Let $(z, \xi)$ belong to the characteristic variety of $M_{\mathfrak{a},-\alpha}$. For a generic $z$, we show $\xi=0$. We follow the proof of [4, Lemma 6].

By $(23)$, we may put $\xi_{\boldsymbol{l} j}=a_{\boldsymbol{l}} b_{j}$ for all $\boldsymbol{l}, j$.

By (24),

$$
0=\sum_{\boldsymbol{l} \in L} z_{\boldsymbol{l}, j_{1}} a_{\boldsymbol{l}} b_{j_{2}}=b_{j_{2}} \sum_{\boldsymbol{l} \in L} z_{\boldsymbol{l}, j_{1}} a_{\boldsymbol{l}}
$$

for all $j_{1}, j_{2} \leq m$.

Suppose that $\xi \neq 0$. Then $\boldsymbol{a} \neq \mathbf{0}$ and $\boldsymbol{b} \neq \mathbf{0}$. Hence

$$
\sum_{\boldsymbol{l} \in L} z_{\boldsymbol{l}, k} a_{\boldsymbol{l}}=0 \quad(k=1, \ldots, m) .
$$

Put $I_{L}:=\left\{\boldsymbol{l} \in L \mid a_{\boldsymbol{l}} \neq 0\right\}$. Then

$$
\sum_{\boldsymbol{l} \in I_{L}} z_{\boldsymbol{l}, k} a_{\boldsymbol{l}}=0 \quad(k=1, \ldots, m) .
$$

Since $z$ is generic, we see

$$
\left|I_{L}\right|>m \text {. }
$$

By (25), we have for $1 \leq k \leq n-2$

$$
0=\sum_{i=0}^{n-k-2} \sum_{j=1}^{m} z_{(i, 0), j} a_{(i+k, 0)} b_{j}=\sum_{i=0}^{n-k-2} a_{(i+k, 0)} c_{(i, 0)}
$$


where we put

$$
c_{(i, 0)}=\sum_{j=1}^{m} z_{(i, 0), j} b_{j} .
$$

Put

$$
I_{L}^{\prime}:=\left\{(k, 0) \mid k=0, \ldots, n-2, a_{(k, 0)} \neq 0\right\}=I_{L} \backslash\{(0,1)\}
$$

and let $k_{0}:=\max \left\{k \mid(k, 0) \in I_{L}^{\prime}\right\}$.

Then we claim

\section{Claim 7.3.}

$$
c_{(k, 0)}=0 \quad \text { for all } k<k_{0} .
$$

Proof. If $k_{0}=0$, then there exists nothing to prove.

Let $k_{0}>0$. We prove the assertion by induction on $k$. Plugging $k=k_{0}$ in $(29)$, we have $a_{\left(k_{0}, 0\right)} c_{(0,0)}=0$. Hence $c_{(0,0)}=0$.

Now we suppose that $c_{(k, 0)}=0$ for all $k<l<k_{0}$. Plugging $k=k_{0}-l$ in $(29)$, we have $a_{\left(k_{0}, 0\right)} c_{(l, 0)}=0$. Hence $c_{(l, 0)}=0$. We have thus proved the claim.

By Lemma 7.1, $(z, \xi)$ satisfies

$$
\alpha_{(n-2,0)} a_{(0,1)} b_{j}=\alpha_{(0,1)} a_{(n-2,0)} b_{j}
$$

for all $1 \leq j \leq m$, and hence we have

$$
\alpha_{(n-2,0)} a_{(0,1)}=\alpha_{(0,1)} a_{(n-2,0)} .
$$

We first treat the case when $\alpha_{(0,1)} \neq 0$.

Suppose that $k_{0}=n-2$. Then $a_{(n-2,0)} \neq 0$, and hence $a_{(0,1)} \neq 0$, i. e. $(0,1) \in I_{L}$, by (31). Then by (27) and (31) we have $\left|I_{L}^{\prime}\right|+1>m+1$, otherwise we have $\boldsymbol{a}=\mathbf{0}$, which is a contradiction. Hence $n>m+1$ or $n-2>m-1$.

By Claim 7.3, we have

$$
c_{(0,0)}=\cdots=c_{(n-3,0)}=0 .
$$

By (30), we have $n-2<m$, otherwise we have $\boldsymbol{b}=\mathbf{0}$, which is a contradiction. Hence $m-1<n-2<m$, which is impossible.

Next suppose that $k_{0}<n-2$. Then $a_{(n-2,0)}=a_{(0,1)}=0$ by $(31)$. By (28), we have $\left|I_{L}^{\prime}\right|>m$, and hence $k_{0}+1>m$.

By Claim 7.3, we have

$$
c_{(0,0)}=\cdots=c_{\left(k_{0}-1,0\right)}=0 .
$$

By (30), we have $k_{0}<m$. Hence $m-1<k_{0}<m$, which is impossible.

We next treat the case when $\alpha_{(0,1)}=0$. Then by (31) we have $a_{(0,1)}=0$. By $(28)$ and the definition of $I_{L}^{\prime}$, we have $\left|I_{L}^{\prime}\right|>m$, and hence $k_{0}+1>m$. 
By Claim 7.3, we have

$$
c_{(0,0)}=\cdots=c_{\left(k_{0}-1,0\right)}=0 .
$$

By (30), we have $k_{0}<m$. Hence $m-1<k_{0}<m$, which is impossible.

Proposition 6.22 implies the form of $g \in N_{G}(\mathfrak{a})$.

\section{Proposition 7.4.}

$$
N_{G}(\mathfrak{a}) / Z_{G}(\mathfrak{a})=\left\{\begin{array}{ll}
{\left[\phi_{l^{\prime}, l}(c)\right]_{l^{\prime}, l \in L_{+}} \mid \begin{array}{l}
c_{(1,0)}^{(1)} c_{(0,1)}^{(2)} \neq 0, \\
c_{(k, 0)}^{(2)}=0
\end{array} \quad(1 \leq k \leq n-3)}
\end{array}\right\} .
$$

Proof. Let $g \in N_{G}(\mathfrak{a}) / Z_{G}(\mathfrak{a})$. Then by Proposition 6.22 we have

$$
g=\left[\phi_{\boldsymbol{l}^{\prime}, \boldsymbol{l}}(c)\right]_{\boldsymbol{l}^{\prime}, \boldsymbol{l} \in L_{+}}
$$

where $c=\left(c_{l}^{(i)}: i=1,2 ; \boldsymbol{l} \in L_{+}\right)$.

We have already seen $c_{(1,0)}^{(1)} \neq 0$ and $c_{(k, 0)}^{(2)}=0(1 \leq k \leq n-3)$ in Example 6.16, and $c_{(1,0)}^{(1)} c_{(0,1)}^{(2)} \neq 0$ in Example 6.18. These are the necessary and sufficient conditions by Proposition 6.22.

Proposition 7.5. Let $\Phi(z ; \alpha)$ be a solution to $M_{\mathfrak{a}, \alpha}$. We assume that a parameter vector

$$
\alpha={ }^{t}\left(\alpha_{(1,0)}, \ldots, \alpha_{(n-2,0)}, \alpha_{(0,1)}\right) \in \mathbb{C}^{L_{+}}
$$

of $\Phi(z ; \alpha)$ satisfies $\alpha_{(n-2,0)} \neq 0$. Then there exists $g \in N_{G}(\mathfrak{a})$ such that the change of coordinates $z \mapsto z^{\prime}=g z$ transforms $\Phi(z ; \alpha)$ into $\Phi\left(z^{\prime} ; \alpha^{\prime}\right)$ with the parameter vector

$$
\alpha^{\prime}={ }^{t}(0, \ldots, 0,1,1) .
$$

Proof. By Lemma 6.6, we have

$$
\begin{aligned}
& \phi_{(i, 0),(0,1)}(c)=c_{(i, 0)}^{(2)} \quad(1 \leq i \leq n-2), \\
& \phi_{(0,1),(j, 0)}(c)= \begin{cases}0 & (j \geq 2) \\
c_{(0,1)}^{(1)} & (j=1),\end{cases} \\
& \phi_{(0,1),(0,1)}(c)=c_{(0,1)}^{(2)} .
\end{aligned}
$$

Then by Proposition 7.4, $g \in N_{G}(\mathfrak{a})$ has the form

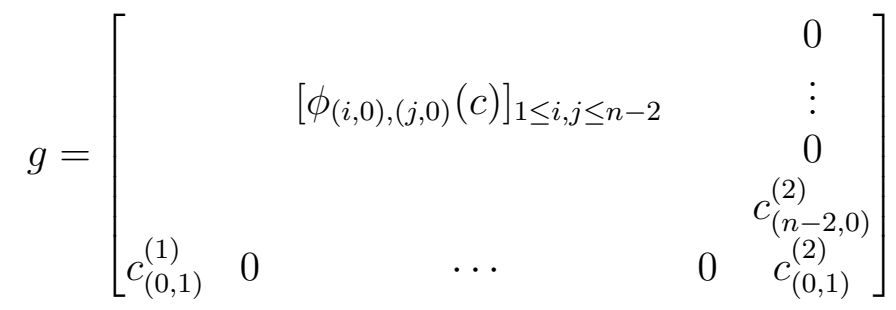


with respect to the order $(1,0), \ldots,(n-2,0),(0,1)$, where $c_{(1,0)}^{(1)} \neq 0$, $c_{(0,1)}^{(2)} \neq 0$ and $c=\left(c_{(1,0)}^{(1)}, \ldots, c_{(n-2,0)}^{(1)}\right) \in \mathbb{C}^{n-2}$.

By (18), we have

$$
\begin{aligned}
& \alpha_{(i, 0)}^{\prime}=\left({ }^{t} g \alpha\right)_{(i, 0)}=\sum_{j=1}^{n-2} \alpha_{(j, 0)} \phi_{i j}(c)+\delta_{i 1} \alpha_{(0,1)} c_{(0,1)}^{(1)}, \\
& \alpha_{(0,1)}^{\prime}=\left({ }^{t} g \alpha\right)_{(0,1)}=\alpha_{(n-2,0)} c_{(1,0)}^{(2)}+\alpha_{(0,1)} c_{(0,1)}^{(2)} .
\end{aligned}
$$

Firstly we consider (32) for $2 \leq i \leq n-2$. Similarly to [8, Corollary $5.4]$, noting that $\alpha_{(n-2,0)} \neq 0$, we can choose $c_{(1,0)}^{(1)}, \ldots, c_{(n-3,0)}^{(1)}$ so that $\alpha_{(2,0)}^{\prime}, \ldots, \alpha_{(n-3,0)}^{\prime}$ become all zero and $\alpha_{(n-2,0)}^{\prime}=1$.

Secondly we consider (32) for $i=1$, i.e.,

$$
\alpha_{(1,0)} c_{(1,0)}^{(1)}+\cdots+\alpha_{(n-2,0)} c_{(n-2,0)}^{(1)}+\alpha_{(0,1)} c_{(0,1)}^{(1)} .
$$

Using the condition $\alpha_{(n-2,0)} \neq 0$, we can determine $c_{(n-2,0)}^{(1)}$ and $c_{(0,1)}^{(1)}$ so that $\alpha_{(1,0)}^{\prime}=0$.

Lastly we consider (33). Noting that $\alpha_{(n-2,0)} \neq 0$, we can determine $c_{(n-2,0)}^{(2)}$ and $c_{(0,1)}^{(2)}$, so that $\alpha_{(0,1)}^{\prime}=1$.

Let $Y=\left(\begin{array}{ccc}y_{01} & \cdots & y_{0 m} \\ \vdots & & \vdots \\ y_{n-2,1} & \cdots & y_{n-2, m}\end{array}\right)$, and define $f: Z \rightarrow Y$ by

$$
f(Z)=\left(\begin{array}{ccc}
z_{(0,0), 1} & \cdots & z_{(0,0), m} \\
\vdots & & \vdots \\
z_{(n-3,0), 1} & \cdots & z_{(n-3,0), m} \\
z_{(n-2,0), 1}+z_{(0,1), 1} & \cdots & z_{(n-2,0), m}+z_{(0,1), m}
\end{array}\right) .
$$

Theorem 7.6. Let $\alpha={ }^{t}(0, \ldots, 0,1,1) \in \mathbb{C}^{n-1}$ and $\beta={ }^{t}(0, \ldots, 0,1) \in$ $\mathbb{C}^{n-2}$. Then

$$
f^{*} M_{(n-1), \beta}=M_{(n-1,1), \alpha} .
$$

Proof. Let

$$
Z^{\prime}=\left(\begin{array}{ccc}
z_{(0,0), 1} & \cdots & z_{(0,0), m} \\
\vdots & & \vdots \\
z_{(n-3,0), 1} & \cdots & z_{(n-3,0), m} \\
z_{(n-2,0), 1}+z_{(0,1), 1} & \cdots & z_{(n-2,0), m}+z_{(0,1), m} \\
z_{(0,1), 1} & \cdots & z_{(0,1), m}
\end{array}\right) .
$$

Then $f=p_{n-1} \circ f^{\prime}$, where $f^{\prime}(Z)=Z^{\prime}$ and $p_{n-1}$ is the projection on the first $n-1$ rows. 
Since $p_{n-1}$ is the projection, we easily see $p_{n-1}^{*} M_{(n-1), \beta}=D_{Z^{\prime}} / J^{\prime}$, where $J^{\prime}$ is generated by

$$
\begin{aligned}
& \frac{\partial}{\partial z_{\left(k_{1}, 0\right), j_{1}}^{\prime}} \frac{\partial}{\partial z_{\left(k_{2}, 0\right), j_{2}}^{\prime}}-\frac{\partial}{\partial z_{\left(k_{1}, 0\right), j_{2}}^{\prime}} \frac{\partial}{\partial z_{\left(k_{2}, 0\right), j_{1}}^{\prime}} \\
& \quad\left(0 \leq k_{1}, k_{2} \leq n-2 ; 1 \leq j_{1}, j_{2} \leq m\right), \\
& \sum_{i=0}^{n-2} z_{(i, 0), j_{1}}^{\prime} \frac{\partial}{\partial z_{(i, 0), j_{2}}^{\prime}}+\delta_{j_{1} j_{2}} \quad\left(1 \leq j_{1}, j_{2} \leq m\right), \\
& \sum_{i=0}^{n-k-2} \sum_{j=1}^{m} z_{(i, 0), j}^{\prime} \frac{\partial}{\partial z_{(i+k, 0), j}^{\prime}}+\beta_{(k, 0)} \quad(k=1, \ldots, n-2), \\
& \frac{\partial}{\partial z_{(0,1), j}^{\prime}} \quad(j=1, \ldots, m) .
\end{aligned}
$$

By the change $f^{\prime}$ of coordinates

$$
\begin{aligned}
z_{(i, 0), j}^{\prime} & =z_{(i, 0), j} \quad(i=0,1, \ldots n-3) \\
z_{(n-2,0), j}^{\prime} & =z_{(n-2,0), j}+z_{(0,1), j} \\
z_{(0,1), j}^{\prime} & =z_{(0,1), j},
\end{aligned}
$$

we have

$$
\begin{aligned}
\frac{\partial}{\partial z_{(i, 0), j}^{\prime}} & =\frac{\partial}{\partial z_{(i, 0), j}} \quad(i=0,1, \ldots n-2) \\
\frac{\partial}{\partial z_{(0,1), j}^{\prime}} & =\frac{\partial}{\partial z_{(0,1), j}}-\frac{\partial}{\partial z_{(n-2,0), j}} .
\end{aligned}
$$

Taking into account that we have

$$
\frac{\partial}{\partial z_{(n-2,0), j}}-\frac{\partial}{\partial z_{(0,1), j}} \in J_{(n-1,1), \alpha}
$$

by Lemma 7.1 , we see $f^{*} M_{(n-1), \beta}={f^{\prime *}}^{*}{ }_{n-1} M_{(n-1), \beta}=M_{(n-1,1), \alpha}$.

Corollary 7.7. Let $\alpha={ }^{t}(0, \ldots, 0,1,1) \in \mathbb{C}^{n-1}$ and $\beta={ }^{t}(0, \ldots, 0,1) \in$ $\mathbb{C}^{n-2}$. Then we have a bijection between the space of solutions to $M_{(n-1,1), \alpha}$ and that to $M_{(n-1), \beta}$.

Proof. Let $f$ be (34). By Theorem 7.6, we have an isomorphism

$$
\begin{aligned}
f^{-1} \operatorname{Hom}_{\mathcal{D}_{Y}}\left(M_{(n-1), \beta}, \mathcal{O}_{Y}\right) & \simeq \operatorname{Hom}_{\mathcal{D}_{Y}}\left(f^{*} M_{(n-1), \beta}, f^{*} \mathcal{O}_{Y}\right) \\
& =\operatorname{Hom}_{\mathcal{D}_{Y}}\left(M_{(n-1,1), \alpha}, \mathcal{O}_{Z}\right) .
\end{aligned}
$$

More explicitly, $\Phi(f(Z))$ is a solution to $M_{(n-1,1), \alpha}$ for a solution $\Phi$ to $M_{(n-1), \beta}$. Conversely any solution to $M_{(n-1,1), \alpha}$ is of this form. 


\section{REFERENCES}

[1] K. Aomoto, Les équations aux différences linéarires et les intégrales des fonctions multiformes, J. Fac. Sci. Univ. Tokyo, Sect. IA Math. 22 (1975) 271-297.

[2] A. G. Elashvili and D. Panyushev, A classification of the principal nilpotent pairs in simple Lie algebras and related problems, J. London Math. Soc. (2) 63 (2001) 299-318.

[3] I. M. Gel'fand, General theory of hypergeometric functions, Dokl. Akad. Nauk. SSSR 288 (1986) 14-18; Soviet Math. Dokl. (English translation), 33 (1987) 9-13.

[4] I. M. Gel'fand, S. I. Gel'fand, Generalized hypergeometric equations, Soviet Mathematics Doklady 33 (1986) 643-646.

[5] I. M. Gel'fand, V. S. Retahk and V. V. Serganova, Generalized Airy functions, Schubert cells and Jordan groups, Dokl. Akad. Nauk. SSSR 298 (1988) 17-21; Soviet Math. Dokl. (English translation), 37 (1988) 8-12.

[6] V. Ginzburg, Principal nilpotent pairs in a semisimple Lie algebra I, Invent. Math. 140 (2000) 511-561.

[7] H. Kimura, Y. Haraoka, K. Takano, On confluences of the general hypergeometric systems, Proc. Japan Acad. 69, Ser. A (1993) 199-104.

[8] H. Kimura, T. Koitabashi, Normalizer of maximal abelian subgroups of GL(n) and general hypergeometric functions, Kumamoto J. Math. 9 (1996) 13-43.

[9] H. Kimura, K. Takano, On confluences of general hypergeometric systems, Tohoku Math. J. 58 (2006) 1-31.

[10] M. Saito, Limits of Jordan Lie subalgebras, J. Lie Theory, 27 (2017) 51-84.

[11] R. Yu, Centralizers of distinguished nilpotent pairs and related problems, J. Algebra 252 (2002) 167-194.

Department of Mathematics, Graduate School of Science, Hokkaido UNIVERSITY, SAPPORO, 060-0810, JAPAN 